\title{
THE CUBIC CHESSBOARD
}

\author{
Richard Kerner \\ Laboratoire de Gravitation et Cosmologie Relativistes \\ Université Pierre et Marie Curie - CNRS, URA 769 \\ Tour 22, 4-ème étage, Boite 142 \\ 4, Place Jussieu, \\ 75005 Paris, France
}

\begin{abstract}
We present a survey of recent results, scattered in a series of papers that appeared during past five years, whose common denominator is the use of cubic relations in various algebraic structures.

Cubic (or ternary relations can represent different symmetries with respect to the permutation group $S_{3}$, or its cyclic subgroup $Z_{3}$. Also ordinary or ternary algebras can be divided in different classes with respect to their symmetry properties. We pay special attention to the non-associative ternary algebra of 3-forms (or "cubic matrices"), and $Z_{3}$-graded matrix algebras.

We also discuss the $Z_{3}$-graded generalization of Grassmann algebras and their realization in generalized exterior differential forms $d \xi$ and $d^{2} \xi$, with $d^{3} \xi=0$. A new type of gauge theory based on this differential calculus is presented.

Finally, a ternary generalization of Clifford algebras is introduced, and an analog of Dirac's equation is discussed, which can be diagonalized only after taking the cube of the $Z_{3}$-graded generalization of Dirac's operator. A possibility of using these ideas for the description of quark fields is suggested and discussed in the last Section.
\end{abstract}


To André Trautman, Teacher and Friend, on the occasion of his $(4)^{3}$-th birthday

\section{Preamble: What I learned from André Trautman}

When the Editors of this Festschrift proposed me to write a contributed paper, I felt not only honored, but also deeply moved by the images of the not so distant past that immediately emerged before my memory's eyes.

Nobody has described this feeling better than Goethe [1]:

Ihr naht euch wieder, schwankende Gestalten, Die früh sich einst dem trüben Blick gezeigt Versuch ich wohl, euch diesmal festzuhalten?

Fühl ich mein Herz nach jenem Wahn geneigt?

Yes, we were all young then, in the Warsaw of the sixties, during the short period between the invention of the gas chambers and man's first steps on the Moon, when things seemed to settle down, maybe not always for the best, but at least in a stable way. They did not settle down for a long time, of course, as it always happens in history. But each generation has to discover that by itself.

To illustrate how young we were then it suffices to say that André at that time was much younger than I am now, although the age difference between us is only eleven years, and he had been freshly promoted to the grade of Professor at the age of 37. Life in Poland was not without shortages then and certainly less easy than in the West. Nevertheless, the basic needs were satisfied in general, and people still remembered that things could be much worse indeed.

Curiously enough, the shortages of culture and science were less acute that those of meat and butter, a somewhat strange situation resulting from strange ideas about human kind ([2], [3], [4]). Polish and Russian books were quite cheap, and most of Western books could be found in the Library of the Institute of Physics of Warsaw University at 69 Hoza street. Also, with good teachers, one could get educated in modern Mathematics and Physics, as adequately as in the best places in the world.

Good teachers we had indeed, and André was among the very best of them: one could learn the brilliance and depth of Mathematics from Krzysztof Maurin, the elegance and beauty of Classical Mechanics and the power of Quantum Field Theory from Iwo Bialynicki-Birula, the universality of Geometry from Wlodzimierz Tulczyjew, and Relativity, Gravitation and Cosmology from André Trautman. I could cite yet many other excellent teachers to whom I owe my first and decisive training in Science.

Although when a few years later I had moved to Paris I had the privilege to continue my education with exceptional teachers like André Lichnerowicz and Yvonne Choquet-Bruhat, this did not alter the fact that at the very foundation, my knowledge is based on what I have been taught during my undergraduate and early graduate years. 
"La culture c'est ce qui reste quand on a tout oublié", said a famous French intellectual - one of the proofs of this statement may be the fact that I am unable to tell now for sure whether it comes from Paul Eluard, Edouard Herriot or André Malraux, - but I still remember the idea!

During the few years between 1964 and 1968, André Trautman taught me the modern and unifying approach to General Relativity and Gauge Theories, expressed by the new means of Differential Geometry: the theory of Fibre Bundles and Connections, cast in an elegant and concise manner with the help of Cartan's exterior calculus [5], [6], [7].

Fibre bundles were constructed as differential manifolds containing both the external and the internal spaces. The so-called external space was the observed four-dimensional space-time, while the internal space was supposed to carry the internal symmetries responsible for the internal conservation laws such as baryonic charge conservation, the isospin conservation, etc. André suggested to me that the five-dimensional theory of Kaluza and Klein in which the fifth dimension is identified with the abelian gauge group $U(1)$ should be generalized to the case of a non-abelian compact Lie group. We would then obtain the lagrangian describing Yang-Mills fields interacting with the gravitational field; the geodesic equation in the fibre bundle would describe the motion of the generalized "colour" charge in an external gauge field ([8], [9], [10], [11]).

Later on, and in the same spirit, I constructed multiple fibre bundles, adding another structural group over a fibre bundle, and so on ([12], [13], [14, [15]). These constructions introduced in a more or less natural way the Higgs fields as "internal" components of generalized gauge fields over a bundle.

In his lectures André always underlined the facts and features that made clear and obvious the continuity of great ideas in science; one could see how the Friedmann-Lemaitre cosmological model could already be constructed in the framework of Newtonian mechanics, with a different interpretation, of course, and with different observational consequences. And when one is aware of the genuine time scale that rules the development of great ideas which stay with us during centuries, one is better prepared to resist the temptation of following short-lived fashions, even when they are declared to be the ultimate and allembracing "theory of everything" (though not accessible to everybody!).

Another thing that impressed me in André's approach to science, and which I tried to follow, was his constant quest for simplicity and elegance. The relations between the phenomena that deserve the name of "laws of Nature" must be simple and expressed by a concise formula ([16]), and one should be able to explain them as basic relations between clear concepts even to a person who has no wide knowledge of physics, because the deeper the concept is, the simpler should be the words and ideas that express it.

As an illustration to this statement I would like to recall a strange feeling of dissatisfaction and presence of a logical flaw, often referred to as the contradictio in adjecto (something nonsensical like "a hot ice cream" or "a giant dwarf"), that often followed me while working with multidimensional generalizations of 
Einstein's theory or gauge-field theories. More than once it occurred to me that it is strange to use the name "internal space" (intended to be an arena of action of the group of internal symmetries giving rise to conservation laws unrelated to the space-time symmetries) to the manifold that is attached as an entity $e x-$ terior to the observable 4-dimensional space-time. One would rather expect the internal degrees of freedom to be encoded inside the external ones; but then the "internal coordinates" should be of an utterly different nature (and probably physical dimension as well) from the usual ones.

That is why later on I was attracted by theories in which the internal degrees of freedom have discrete character, like e.g. the fermionic "coordinates" in supersymmetry, or even more radically different as in the case of non-commutative geometries, in which the very notion of a manifold is no longer relevant. Together with Michel Dubois-Violette and John Madore we have produced some contributions in this direction, published in a series of papers in 1989, 1990 and 1991; important works in this area by Alain Connes, John Lott, Robert Coquereaux and others are by now widely known ([17], [18], [19], [22], [20], [21]).

Since the end of the year 1990, I have started to think about non-associative and ternary generalizations of supersymmetry and the non-commutative geometries. Here again, I was led by the simple intuition that the smaller the scale, the stronger the interactions, so that the geometry at very small scales should be governed by the strongest interactions we know, which are apparently the strong interactions described by quark models.

Who knows if the magic number 3 that seems to govern the world of quarks is not related to the dimension 3 of the space we are living in ? If so, it might be worthwhile to study carefully the mathematical structures that particular

ternary symmetries display, based on the groups $Z_{3}$ and $S_{3}$ (resp. cyclic and general permutations of 3 objects) instead of $Z_{2}$ (see 25]).

In what follows, I shall develop some arguments in favor of this new approach, and show a few examples of its realization and consequences.

\section{Motivation for the study of ternary algebras}

One of the most visible logical threads that is constantly present in modern theoretical physics dealing with fundamental interactions is the unification. idea. Well known examples of this way of thinking are provided by:

a) Unified theories of Kaluza-Klein type, including the standard model of electroweak interactions by Weinberg and Salam, which finds its mathematical expression in the theory of connections and curvature on fibre bundles;

b) Supersymmetric theories, superposing and unifying the fields corresponding to particles of integer and half-integer spin obeying different quantum statistics, their mathematical framework being provided by the theory of superalgebras and analysis on supermanifolds;

c) Quantum scattering problems and mathematical extension of group theory to quantum groups (Hopf algebras). 
The search for unification is intimately related to the idea of spontaneous symmetry breaking, consisting of the conviction that we usually observe only a partial and reduced symmetry instead of a more complete and full symmetry that might exist among the states of the system under consideration.

It seems possible to extend this approach of unifying symmetries to the algebraic structure itself, by superposing the categories of linear spaces and the spaces of (multi) linear mappings of tensor powers of these spaces into themselves. One of the best ways to illustrate our ideas and to motivate the interest in 3-algebras is to analyze the theory of supersymmetry from a slightly new point of view.

The basic ingredient of supersymmetry is the introduction of the possibility of mixing and to superposing linearly the states corresponding to pure fermions or bosons, which is believed to be impossible at the energies we have at our disposal up to now. In this sense supersymmetry restores the broken symmetry, under which the allowed transformations should never mix the fields of integer spin with those of half-integer spin. It is supposed that at least on some yet unexplored energy scale the so-called superfields may appear, constructed from fields belonging to all possible irreducible representations of the Lorentz group, with coefficients belonging to the Grassmann algebra generated by a set of anti-commuting spinors:

$$
\Phi\left(x^{\mu}, \theta^{\alpha}, \bar{\theta}^{\dot{\beta}}\right):=\phi_{o}\left(x^{\mu}\right)+\psi_{\alpha}(x) \theta^{\alpha}+\bar{\theta}^{\dot{\beta}} \bar{\psi}_{\dot{\beta}}+W_{\mu} \sigma_{\alpha \dot{\beta}}^{\mu} \theta^{\alpha} \bar{\theta}^{\dot{\beta}}+\ldots
$$

The "superfield" is thus a multiplet of fields that contains a scalar $\phi_{o}$, a four-vector $W_{\mu}$, a Majorana spinor $\psi_{\alpha}$ and its conjugate $\bar{\psi}_{\dot{\beta}}$, etc.; here $\theta^{\alpha}$ and $\bar{\theta}^{\dot{\beta}}$ are anti-commuting variables that span the Grassmann algebra, and who behave like Majorana spinors with respect to Lorentz transformations.

Let us note at this point that spinors can be viewed as fundamental quantities here, whereas both scalar and 4-vector fields can be found as irreducible representations in the tensor product of spinors, following the classical decomposition formula for the representations of the Lorentz group:

$$
D^{\left(\frac{1}{2}, 0\right)} \otimes D^{\left(0, \frac{1}{2}\right)}=D^{(1,0)} \oplus D^{\left(\frac{1}{2}, \frac{1}{2}\right)}
$$

Prior to this decomposition, any tensor belonging to the product $D^{\left(\frac{1}{2}, 0\right)} \otimes D^{\left(0, \frac{1}{2}\right)}$ can be identified with a linear operator acting on the space of spinors. This is a particular case of a general correspondence existing between the linear operators on a Hilbert space and a tensor square of this space:

$$
\text { Let } x, y \in V, \quad A \in L(V, V) \text {, and let } A(x)=y
$$

where $V$ is a Hilbert space, and $A$ a linear operator defined on it.

Then in a given orthonormal basis $\left\{e_{i}\right\}$, with the usual identification of the dual basis (i.e. the basis of linear functionals on $V$ ) by means of a scalar product in $H: e^{* k}\left(e_{i}\right)=\left\langle e_{k}, e_{i}\right\rangle=\delta_{i}^{k}$, we may write $A \sim \sum_{k, i} A_{i}^{k} e_{k} e^{* i}$, so that 


$$
A(x)=\sum_{k, i} A_{i}^{k} e_{k} \otimes e^{* i}\left(\sum_{m} x^{m} e_{m}\right)=\sum_{k, m} A_{m}^{k} x^{m} e_{k}=y^{k} e_{k}=y
$$

The linear operator $A$ defined by its matrix elements $A_{m}^{k}$ can be also identified with the element of the tensor product $V \otimes V$ as

$$
A \Rightarrow \sum_{k, m} A_{k m} e_{k} \otimes e_{m}, \text { with } A_{k m}:=A_{m}^{k}
$$

In the above picture the rôle of the two copies of the Hilbert space that have been used to produce general linear operators (containing the algebra of observables) by means of the tensor product is utterly different from the rôle of the third copy serving as the space of states, as may be seen below:

$$
(L(V, V) \oplus V) \sim(V \otimes V) \oplus V
$$

In some sense it is also analogous with a strange and unusual summation that is performed on the indices of the Riemann tensor in order to obtain the Ricci tensor: let us recall that although all the indices of the Riemann tensor vary within the same range, which is the dimension of the Riemannian manifold $V_{n}$ on which the metric and the Riemann tensor are defined, their nature is totally different. The curvature, which is a Lie algebra valued 2-form, can be written in a given coordinate system as:

$$
\Omega=\Omega_{i j} d x^{i} \wedge d x^{j}=R_{i j m}^{k} E_{k}^{m} d x^{i} \wedge d x^{j}
$$

where the matrices $E_{k}^{m}$ span the basis of the $n^{2}$-dimensional Lie algebra of the linear group $G L_{n}(\mathrm{R})$, satisfying the commutation relations

$$
E_{k}^{m} E_{l}^{i}-E_{l}^{i} E_{k}^{m}=\delta_{l}^{m} E_{k}^{i}-\delta_{k}^{i} E_{l}^{m}
$$

Here again, the matrices $E_{k}^{m}$ can be put in a one-to-one correspondence (by lowering one of the indices with the metric tensor $g_{l m}, E_{k}^{m} \rightarrow g_{l m} E_{k}^{m}=E_{l k}$ ) with the elements of the tensor product of 1 -forms, $V^{*} \otimes V^{*}$ In the definition of the Ricci tensor, $R_{i k}=\sum_{j} R_{i j k}^{j}$, we perform the summation over the indices belonging to different realms: one comes from the vector space, while another is a part of the multi-index labeling the elements of the space of linear transformations (matrices) acting on this space.

In some sense these examples look like a discrete version of the choice of a local section in a fibre bundle, whose total space would be $V \otimes V \otimes V$.

This suggests that one could restore the full symmetry between the three copies of the vector space $V$ by embedding $L(V, V) \oplus V$ into the tensor cube $V \otimes V \otimes V$, which can be then "reduced" or "projected" in six different ways onto $L(V, V) \oplus V$.

An arbitrary element $a \in V \otimes V \otimes V$ can be written in the basis $\left\{e_{k}\right\}$ as

$$
a=\sum_{k, l, m} a_{k l m} e_{k} \otimes e_{l} \otimes e_{m}
$$


which defines a one-to-one correspondence between the elements of the space $V \otimes V \otimes V$ and the three-tensors $a_{k l m}$, which we will call also cubic matrices from now on.

It might be that one of the reasons for the non-observability of quarks is related to the fact that they belong to this kind of "mixed" space, in which there is no clear distinction between the state and the observable, both being included in a 3-form (or a "cubic matrix").

The symmetric group $S_{3}$ (of permutations of three elements) acts in an obvious way on the complex cubic matrices by permuting their indices. It is therefore natural to distinguish separate subspaces of $V \otimes V \otimes V$ that provide the irreducible representations of $S_{3}$; e.g., there is the subspace of the totally symmetric cubic matrices satisfying $a_{k l m}=a_{l m k}=a_{m l k}=\ldots$, the subspace of $j$-skew-symmetric cubic matrices satisfying $b_{k l m}=j b_{l m k}=j^{2} b_{m l k}=b_{m l k}^{-}$, etc.

Curiously enough, the most natural internal composition law that generalizes the multiplication of ordinary matrices (or of the elements of $V \otimes V$ ) is ternary and is given by the following rule:

$$
(a \oslash b \oslash c)_{i j k}:=\sum_{p, q, r} a_{i p q} b_{p j r} c_{q r k}
$$

In contrast with ordinary matrix multiplication this composition is nonassociative, in the sense that

$$
(a \oslash(b \oslash c \oslash d) \oslash e) \neq(a \oslash b \oslash c) \oslash d \oslash e \neq a \oslash b \oslash(c \oslash d \oslash e)
$$

Note that the group $S_{3}$ acts also on the so defined ternary algebra, so that both actions (the permutation of factors in the product and the permutation of the indices in the resulting cubic algebra) can compensate each other thus defining invariant classes in our algebra.

Such ternary products have been introduced in ([25] and [26], and studied also by R. Lawrence (27]) and L. Vainerman and the author (28]), and are, in fact, a particular case of a more general $n$-fold multiplication defined on the $n$-tensors as follows:

$$
\begin{gathered}
m\left(a^{(1)}, a^{(2)}, \ldots, a^{(n)}\right)_{i_{1} i_{2} \ldots i_{n}}=\sum_{j_{k r}=1(k<r)}^{l} a_{i_{1} j_{12} \ldots j_{1 n}}^{(1)} a_{j_{12} i_{2} j_{23} \ldots j_{2 n}}^{(2)} \times \ldots \times \\
\times a_{j_{1 k} \ldots j_{k-1 k} i_{k} j_{k k+1} \ldots j_{k n}}^{(k)} \times \ldots \times a_{j_{1 n} \ldots j_{n-1 n} i_{n}}^{(n)}
\end{gathered}
$$

We believe that the ternary case is exceptional because it involves the symmetry group $S_{3}$ (permutations of three objects, or indices), and this group is the last one that possesses an exact and faithful representation in the field of complex numbers, the next one, $S_{4}$, has a representation with a double degeneracy, while starting from $S_{5}$ there are no representations in $\mathbf{C}$. 


\section{The 3-algebra of cubic matrices: the cubic chessboard}

Let us concentrate now on a more detailed study of the ternary algebra of complex-valued cubic matrices. Such a study seems to be particularly important in view of the pertinence of these matrices (which are isomorphic with the elements of $V \otimes V \otimes V)$ to a possible generalization of quantum mechanics and field theory.

Also, from a purely mathematical point of view, a ternary composition law

in a linear space over complex numbers is particularly interesting because it can be decomposed in an irreducible way with respect to the permutation group $S_{3}$, which is the last of the permutation groups that has a faithful representation in the complex plane ([30]). Later on we shall give further arguments suggesting the exceptional rôle of cubic matrices and their ternary algebra; for the time being, it suffices to draw attention to the fact that the cubic matrices can be visualized in three dimensions (like the "Rubik's cube", for example), and they are probably the last case that can be still treated and analyzed in a finite time, although even in this case the use of the computer becomes crucial.

We start by fixing the notation and conventions. Let the indices $i, k, l, m, \ldots$ run from 1 to $N$. Let the elements of a (complex-valued) matrix $a$ in a given basis be $a_{i k m}$. The multiplication introduced previously is defined as:

$$
(a \oslash b \oslash c)_{i j k}:=\sum_{p, q, r} a_{i p q} b_{p j r} c_{q r k}
$$

The ternary "multiplication table" is like a cubic chessboard with dimensions $\left(N^{3}\right) \times\left(N^{3}\right) \times\left(N^{3}\right)$, which amounts to 512 different entries for the case $N=2$ and to $27^{3}=3^{9}=19683$ different entries for the case $N=3$ - some chessboard, indeed! That is why in what follows we shall restrain ourselves to the cases $N=2$ and $N=3$ only.

One of the natural bases in the space of cubic matrices is the set defined as:

$e_{i k m}:=1$ at the intersection of $i$-th, $k$-th and $m$-th rows, 0 elsewhere

Let us show that in the case $N=2$ it is possible to obtain a decomposition of the 8-dimensional ternary algebra (as a linear space) into the direct sum of its three special subalgebras. In fact there are 8 matrix units in the whole algebra. Three of them: $e_{111}, e_{222}, e_{333}$ generate a subalgebra Diag of the diagonal matrices which is evidently $S_{n}$-commutative. Using the ternary multiplication formula for the considered partial case, one can compute that the subalgebra generated by the matrix units $e_{112}, e_{121}, e_{122}$ has a zero multiplication and consequently it is abelian. The same is true for the subalgebra generated by the matrix units $e_{221}, e_{212}, e_{211}$. Thus, we have a decomposition

$$
\operatorname{Mat}(2,3 ; C)=\operatorname{Diag} \oplus\left\{e_{112}, e_{121}, e_{122}\right\} \oplus\left\{e_{221}, e_{212}, e_{211}\right\},
$$

in which the first summand is $S_{n}$-commutative and the two others are abelian subalgebras. This decomposition looks like a decomposition of $2 \times 2$-matrices on the diagonal and two triangular subalgebras. But it is not unique; one can 
get at least two similar decompositions:

$$
\begin{gathered}
\operatorname{Mat}(2,3 ; C)=\operatorname{Diag} \oplus\left\{e_{112}, e_{212}, e_{211}\right\} \oplus\left\{e_{121}, e_{122}, e_{221}\right\} \text { and } \\
\operatorname{Mat}(2,3 ; C)=\operatorname{Diag} \oplus\left\{e_{121}, e_{221}, e_{211}\right\} \oplus\left\{e_{112}, e_{122}, e_{212}\right\} .
\end{gathered}
$$

But it is another decomposition, connected with the representation properties with respect to the group $Z_{3}$ (eventually $S_{3}$ ) that will be important in our forthcoming study of ternary algebra of cubic matrices.

Let $J$ be the cyclic permutation operator acting on cubic matrices as:

$$
(J a)_{i k l}:=a_{k l i} ; \text { obviously }\left(J^{2} a\right)_{i k l}:=a_{l i k}, \quad \text { and } J^{3}=I d
$$

In the articles ([25], [28]) we have introduced an alternative multiplication law for cubic matrices defined below:

$$
(a * b * c)_{i k l}:=\sum_{p q r} a_{p i q} b_{q k r} c_{r l p}
$$

in which any cyclic permutation of the matrices in the product is equivalent to the same permutation on the indices:

$$
(a * b * c)_{i k l}=(b * c * a)_{k l i}=(c * a * b *)_{l i k}
$$

It is easy to see that the two multiplication laws are related as follows:

$$
a \oslash b \oslash c=(J a) * b *\left(J^{2} c\right)
$$

and neither of the two is associative. Let us denote by $j$ the cubic root of unity, $j=e^{\frac{2 \pi i}{3}}$; we have $j+j^{2}+1=0$, and $\bar{j}=j^{2}$.

The complex square $N \times N$-matrices can be divided into subspaces with particular representation properties with respect to the group of permutations $S_{2}$ (isomorphic with $Z_{2}$ ), thus defining symmetric, anti-symmetric, hermitian and anti-hermitian matrices: let $T$ be the transposition operator, $(T a)_{i k}=a_{k i}$; then we can define the aforementioned types of matrices as the ones that have the following transformation laws under the action of $T$ :

$$
T a=a, \quad T a=-a, \quad T a=\bar{a}, \quad T a=-\bar{a}
$$

which gives in index notation the usual definitions:

$$
a_{i k}=a_{k i}, \quad a_{i k}=-a_{k i}, \quad a_{i k}=\bar{a}_{k i}, \quad a_{i k}=-\bar{a}_{k i},
$$

Similarly, the complex cubic matrices can be divided into classes according to the representations of the group $S_{3}$. With $J$ defined as above (9) and $T$ the operator of odd transposition, $(T a)_{i k m}=a_{m k i}$, we can define cubic matrices with the following non-equivalent representation properties under the action of the operators $J$ and $T$ :

$$
J a=a, \quad T a=a ; \quad J a=j a, \quad T a=a ; \quad J a=j^{2} a, \quad T a=a
$$


and

$$
J a=a, \quad T a=\bar{a} ; \quad J a=j a, \quad T a=\bar{a} ; \quad J a=j^{2} a, \quad T a=\bar{a}
$$

From now on we shall concentrate on the class of matrices displaying welldefined properties with respect to the group of cyclic permutations $Z_{3}$ only, i.e. supposing that there is no particular relation between $a$ and $T a$.

This type of decomposition is important in the analysis of the possible representations of ternary algebras of cubic matrices in terms of associative matrix algebras. We shall follow the well known example of Ado's theorem for finitedimensional Lie groups, which states that for such groups an associative enveloping algebra can be found, such that the skew-symmetric, non-associative composition law satisfying the Jacobi identity can be faithfully represented by a commutator of the corresponding elements.

Although at this stage we are don't know if an analogue of the Jacobi identity exists for ternary algebra of cubic matrices, we shall show that at least for the simplest cases, certain ternary algebras with a non-associative composition law displaying particular symmetries can be represented in the algebra of associative matrices. Let us decompose the algebra of cubic matrices into the direct sum of the following linear subspaces:

Diagonal, containing $\mathrm{N}$ diagonal cubic matrices $\omega^{(k)}$ :

$$
\omega_{k k k}^{(k)}=1 \text {, all other elements }=0 ;
$$

Symmetric, containing $\left(N^{3}-N\right) / 3$ traceless, totally symmetric cubic matrices

$$
\pi_{k l m}^{(\alpha)}=\pi_{l m k}^{(\alpha)}=\pi_{m k l}^{(\alpha)}, \quad \alpha=1,2, . .,\left(N^{3}-N\right) / 3
$$

j-Skew-symmetric, containing $\left(N^{3}-N\right) / 3$ cubic matrices satisfying

$$
\rho_{k l m}^{(\alpha)}=j \rho_{l m k}^{(\alpha)}=j^{2} \rho_{m k l}^{(\alpha)}
$$

and $j^{2}$-Skew-symmetric, containing $\left(N^{3}-N\right) / 3$ cubic matrices satisfying

$$
\kappa_{k l m}^{(\alpha)}=j^{2} \kappa_{l m k}^{(\alpha)}=j \kappa_{m k l}^{(\alpha)} .
$$

Only the diagonal matrices form a 3-subalgebra with respect to the ternary multiplication law;

$$
\omega^{(k)} * \omega^{(l)} * \omega^{(m)}=0 \text { if } \quad k \neq l \neq m \quad \text { and } \omega^{(k)} * \omega^{(k)} * \omega^{(k)}=\omega^{(k)}
$$

This 3-subalgebra is associative and commutative and is easily represented by ordinary (square) matrices $\omega^{(k)}$, whose only non-vanishing element 1 is found at the intersection of the $\mathrm{k}$-th line with the $\mathrm{k}$-th column.

With eight independent generators the ternary algebra's multiplication table is also a cubic array, and in order to define it completely we must display as many as $8 \times 8 \times 8=512$ different ternary products. Because of the non-associativity of ternary law, it is impossible to find a realization of these multiplication rules by means of a set of finite $n \times n$ matrices.

This situation is not new indeed, and could be observed in the case of binary non-associative algebras. The well known Ado's theorem states that a 
class of finite dimensional non-associative algebras with particular symmetry of the composition law, $\{X, Y\}=-\{Y, X\}$ and satisfying the Jacobi identity (Lie algebras) can always be represented by a subset of some bigger associative algebra, called the enveloping algebra.

Let us show on a simple example of $2 \times 2 \times 2$ cubic matrices that a representation in the associative binary algebra of $2 \times 2$ ordinary matrices can be found provided that the ternary composition law is endowed with a particular symmetry that generalizes the skew symmetry of the ordinary Lie algebra.

In the multiplication table for the cubic matrices in the particular basis of $\omega^{(k)}, \pi^{(\alpha)}, \rho^{(\beta)}$ and $\kappa^{(\gamma)}$ it is difficult to find any subalgebras except for the obvious "central" one containing the $\omega^{(k)}$. Usually a 3-product of three matrices will decompose into a linear combination of the matrices belonging to various symmetry types, e.g.

$$
\rho^{(1)} * \rho^{(1)} * \rho^{(2)}=\omega^{(1)}-\frac{1}{3} \pi^{(2)}+\frac{2}{3} j^{2} \rho^{(2)}-\frac{1}{3} j \kappa^{(2)}, \text { etc. }
$$

The situation changes if we introduce a new composition law that follows the particular symmetry of the given type of cubic matrices. For example, let us define:

$$
\left\{\rho^{(\alpha)}, \rho^{(\beta)}, \rho^{(\gamma)}\right\}:=\rho^{(\alpha)} * \rho^{(\beta)} * \rho^{(\gamma)}+j \rho^{(\beta)} * \rho^{(\gamma)} * \rho^{(\alpha)}+j^{2} \rho^{(\gamma)} * \rho^{(\alpha)} * \rho^{(\beta)}
$$

Because of the symmetry of the ternary $j$-bracket one has

$$
\left\{\rho^{(\alpha)}, \rho^{(\beta)}, \rho^{(\gamma)}\right\}_{i k m}=j\left\{\rho^{(\alpha)}, \rho^{(\beta)}, \rho^{(\gamma)}\right\}_{k m i},
$$

so that it becomes obvious that with respect to the $j$-bracket composition law the matrices $\rho^{(\alpha)}$ form a ternary subalgebra. Indeed, we have

$$
\left\{\rho^{(1)}, \rho^{(2)}, \rho^{(1)}\right\}=-\rho^{(2)} ; \quad\left\{\rho^{(2)}, \rho^{(1)}, \rho^{(2)}\right\}=-\rho^{(1)} ;
$$

all other combinations being proportional to the above ones with a factor $j$ or $j^{2}$, whereas the j-brackets of three identical matrices obviously vanish.

Our aim is to find the simplest representation of this ternary algebra in terms of a $j$-commutator defined in an associative algebra of matrices $M_{2}(C)$ as follows:

$$
[A, B, C]:=A B C+j B C A+j^{2} C A B
$$

It is easy to see that the trace of any j-bracket of three matrices must vanish; therefore, the matrices that would represent the cubic matrices $\rho^{(\alpha)}$ must be traceless. Then it is a matter of simple exercise to show that any two of the three Pauli sigma-matrices divided by $\sqrt{2}$ provide us with a representation of the ternary j-skew algebra of the $\rho$-matrices; e.g.

$\sigma^{1} \sigma^{2} \sigma^{1}+j \sigma^{2} \sigma^{1} \sigma^{1}+j^{2} \sigma^{1} \sigma^{1} \sigma^{2}=-2 \sigma^{2}, \quad \sigma^{2} \sigma^{1} \sigma^{2}+j \sigma^{1} \sigma^{2} \sigma^{2}+j^{2} \sigma^{2} \sigma^{2} \sigma^{1}=-2 \sigma^{1}$

Thus, it is possible to find a representation in the associative algebra of finite matrices for the non-associative j-bracket ternary algebra. A similar representation can be found for the two cubic matrices $\kappa^{(\alpha)}$ with the $j^{2}$-skew bracket. 
If there exists an analogue of the Jacobi identity, it cannot contain the double j-brackets. As a matter of fact, we have been able to prove that there are no non-trivial solutions to the equation containing the forty non-redundant double j-brackets like $[A,[B, C, D], E]$. Therefore, in order to produce a non-trivial analogue of the Jacobi identity for ternary algebras, we should find an identity involving seven different entities, like

$$
[A,[B,[C, D, E], F] G] \text {, and }[[A, B, C] D[E, F, G]] \text {, etc. }
$$

It is also worthwhile to note that the ordinary Lie algebras with the skewsymmetric composition law can be found in the representation of the ternary $\mathrm{j}$-bracket algebra in the associative algebra, provided the latter one is endowed with a central (unit) element. Indeed, we have:

$$
[A, \mathbf{1}, C]=A \mathbf{1} C+j \mathbf{1} C A+j^{2} C A \mathbf{1}=A C+\left(j+j^{2}\right) C A=A C-C A
$$

The fact that Pauli matrices did appear in a quite natural way is encouraging. It suggests that although we start here from a ternary algebra with $j$-skew 3 -commutator, more familiar notions such as the Lorentz group and spin can be encoded in some way in this unusual rules, and appear sooner or later as secondary features of a purely algebraic theory.

The following exercise reinforces this hope.

A natural question to ask now concerns the nature of all the automorphisms of this simple ternary algebra. The most general homogeneous transformation of the cubic matrices $\rho^{(\alpha)}$ involves all their indices:

$$
\tilde{\rho}_{i k m}^{(\alpha)}=\Lambda_{\beta}^{\alpha} U_{i}^{p} U_{k}^{r} U_{m}^{s} \rho_{p r s}^{(\beta)}, \quad \alpha, \beta, i, k, \ldots=1,2 .
$$

with (invertible) matrices $\Lambda_{\beta}^{\alpha}, U_{i}^{p}$ chosen in such a way that the ternary relations between the transformed cubic matrices $\tilde{\rho}^{(\alpha)}$ remain the same as defined above.

Let us show that even in a simplified case when we choose $U_{q}^{p}=\delta_{q}^{p}$, the condition of invariance of the ternary algebra leads to non trivial solutions for the group of matrices $\Lambda_{\beta}^{\alpha}$. As a matter of fact, we get the following system of equations for $\Lambda_{\beta}^{\alpha}$ :

$$
\begin{array}{cc}
\Lambda_{1}^{1}\left(\Lambda_{2}^{2} \Lambda_{1}^{1}-\Lambda_{2}^{1} \Lambda_{1}^{2}\right)=\Lambda_{2}^{2} ; & \Lambda_{2}^{1}\left(\Lambda_{1}^{2} \Lambda_{2}^{1}-\Lambda_{1}^{1} \Lambda_{2}^{2}\right)=\Lambda_{1}^{2}, \quad \text { and } \\
\Lambda_{2}^{2}\left(\Lambda_{1}^{1} \Lambda_{2}^{2}-\Lambda_{1}^{2} \Lambda_{2}^{1}\right)=\Lambda_{1}^{1} ; & \Lambda_{1}^{2}\left(\Lambda_{2}^{1} \Lambda_{1}^{2}-\Lambda_{2}^{2} \Lambda_{1}^{1}\right)=\Lambda_{2}^{1}
\end{array}
$$

from which follows that $[\operatorname{det}(\Lambda)]^{2}=1$, so that either

$$
\begin{gathered}
\operatorname{det}(\Lambda)=1, \quad \text { and } \quad \Lambda_{1}^{1}=\Lambda_{2}^{2}, \quad \Lambda_{2}^{1}=-\Lambda_{1}^{2}, \quad \text { or } \\
\operatorname{det}(\Lambda)=-1, \quad \text { and } \quad \Lambda_{1}^{1}=-\Lambda_{2}^{2}, \quad \Lambda_{2}^{1}=\Lambda_{1}^{2} .
\end{gathered}
$$

This group has two disjoint components; the simply connected component of the unit element is a subgroup, whereas the second component can be obtained from the first one by multiplication by the $2 \otimes 2$ matrix $\operatorname{diag}(1,-1)$. 
The simply connected subgroup is an abelian, (real) two-dimensional Lie group of matrices whose general form is

$$
\left(\begin{array}{cc}
a & b \\
-b & a
\end{array}\right) \text {, with } a, b \text { complex numbers satisfying } a^{2}+b^{2}=1
$$

which can be decomposed into a simple product of two matrices:

$$
\left(\begin{array}{cc}
\cosh \psi & i \sinh \psi \\
-i \sinh \psi & \cosh \psi
\end{array}\right)\left(\begin{array}{cc}
\cos \phi & \sin \phi \\
-\sin \phi & \cos \phi
\end{array}\right)
$$

This group is easily identified as the simple product of Euclidean rotations and translations. It can be realized as the isometry group of a cylindrical Minkowski space parametrised with two variables $\tau$ and $\phi,[0 \leq \phi \leq$ $2 \pi] \times[-\infty \leq \tau \leq \infty]$, with one "boost" and one angular translation. When embedded in a many-dimensional Minkowski space, this object looks like a motionless closed string. This invariance group reduces to $U(1)$ if we impose the reality condition on the matrices $\rho(\alpha)$ requiring that $\rho_{i k l}^{(\alpha)}=\bar{\rho}_{l k i}^{(\alpha)}$.

The ternary algebra of complex cubic matrices in three dimensions, Mat $(3,3, C)$, has a very rich structure; its multiplication table (in three dimensions, too) can be visualized as a cubic matrix with $27 \times 27 \times 27=3^{9}$ entries. Here again, subsets displaying a particular $Z_{3}$-symmetry can be defined, containing eight independent matrices each, so that the whole algebra of 27 independent matrices decomposes as $M=\operatorname{Diag} \oplus M_{0} \oplus M_{1} \oplus M_{2}$.

Let us denote these cubic matrices by: $O_{b c d}^{(a)}$ (the diagonal part); $R_{a b c}^{(A)}$, with $A=1,2, \ldots 8$, and $a, b=1,2,3$ spanning the subset $M_{1} ; K_{a b c}^{(A)}$ spanning the subset $M_{2}$, and $P_{a b c}^{(A)}$ spanning the totally $Z_{3}$-symmetric traceless subset $M_{0}$. The cubic matrices denoted by capital Latin letters display the same symmetries as their prototypes belonging to $\operatorname{Mat}(2,3, C)$ denoted by the corresponding Greek letters $\omega, \rho, \kappa$ and $\pi$. It is easy to see that the component $M_{1}$ containing the matrices $R^{(A)}$ satisfying

$$
R_{a b c}^{(A)}=j R_{b c a}^{(A)}=j^{2} R_{c a b}^{(A)}
$$

consists of three two-dimensional ternary subalgebras, each of them isomorphic with the algebra of $\rho$-matrices shown above. The three subalgebras are spanned by (we just give the only non-vanishing elements):

$$
\left\{R_{232}^{(1+)}, R_{323}^{(1-)}\right\} ; \quad\left\{R_{313}^{(2+)}, R_{131}^{(2-)}\right\} \text { and }\left\{R_{121}^{(3+)}, R_{212}^{(3-)}\right\}
$$

besides, there are two more independent generators,

$$
R_{123}^{(7)} \text { and } R_{321}^{(8)}
$$

This situation is similar to the one observed in the examples of the Lie algebras $s u(2)$ and $s u(3)$, where the algebra $s u(2)$ can be embedded in three different ways in the algebra $s u(3)$. It is also clear that among the automorphisms of the ternary algebra spanned by $R^{(A)}$, with the $j$-skew ternary commutator, we will find three copies of the automorphisms of the simple ternary algebra of $\rho^{(\alpha)}$ 
cubic matrices, which means that we shall have three independent Lorentzian boosts, and three independent rotations of a plane, which is exactly what is needed to generate the 6-parameter Lorentz group in 4-dimensional space-time. Similar observation can be made concerning the cubic matrices $K^{(A)}$.

This does not exclude the possibility of finding other interesting subgroups in the group of automorphisms of ternary relations between the cubic matrices $R^{(A)}$ or $K^{(A)}$, e.g. the group $S U(3)$ in its adjoint representation, althoughit may be intertwined with the elements of the Lorentz group in a very tricky way.

To find a maximal ternary subalgebra of $M_{1} \subset \operatorname{Mat}(3,3 ; C)$ that can be represented in a finite associative algebra with the $j-s k e w$ commutator as the composition law is not an easy task, and we don't know the full answer to this problem. However, the fact that the traceless part of the 3-algebra of cubic matrices splits naturally into three equal parts suggests that its representation by means of an associative eneveloping algebra can be naturally $Z_{3}$-graded, with three grades $0,1,2$ adding up modulo 3 . Such algebras are also very interesting, and we were able to investigate them to some extent; some of the results are presented in the following Sections.

\section{4. $Z_{3}$-graded associative algebras}

The simplest case of an associative $Z_{3}$-graded algebra is provided by the algebra of complex $3 \times 3$ matrices, which is divided into the following three linear subspaces: $\mathcal{M}_{3}(C)=\mathcal{A}_{0} \oplus \mathcal{A}_{1} \oplus \mathcal{A}_{2}$, with

$$
\left(\begin{array}{ccc}
\alpha & 0 & 0 \\
0 & \beta & 0 \\
0 & 0 & \gamma
\end{array}\right) \in \mathcal{A}_{0} ; \quad\left(\begin{array}{ccc}
0 & \alpha & 0 \\
0 & 0 & \beta \\
\gamma & 0 & 0
\end{array}\right) \in \mathcal{A}_{1} ; \quad\left(\begin{array}{ccc}
0 & 0 & \gamma \\
\alpha & 0 & 0 \\
0 & \beta & 0
\end{array}\right) \in \mathcal{A}_{2} \quad \alpha, \beta, \gamma \in C \text {. }
$$

One easily checks that if the elements $a^{(k)}$ and $b^{(m)}$ belong to the subspaces $\mathcal{A}_{k}$ and $\mathcal{A}_{m}$ respectively, $(k, m=0,1,2)$, then their matrix product belongs to $\mathcal{A}_{(k+m) \bmod (3)}$. This algebra may be viewed upon as a model of non-commutative $Z_{3}$-graded geometry, generalizing the $Z_{2}$-graded matrix algebra used in the models of elementary interactions based on the noncommutative $Z_{2}$-graded geometry, ([17], [18], [19], [20], [21], [23], [24]).

Let us introduce the following $Z_{3}$-graded commutator:

$$
[A, B]_{Z_{3}}=A B-j^{a b} B A, \quad \text { with } a=\operatorname{grad}(A), \quad b=\operatorname{grad}(B) .
$$

Denoting the $Z_{3}$-commutator of element $A$ with any other element $B$ by $\operatorname{Der}_{A}(B)$, (both of them having the well defined $Z_{3}$-grade), it is easy to check the $Z_{3}$-graded Leibniz rule in $\mathcal{A}$ :

$$
\operatorname{Der}_{A}(B C)=\left[\operatorname{Der}_{A}(B)\right] C+j^{a b} B\left[\operatorname{Der}_{A}(C)\right]
$$

However, these derivations do not form a $\left(Z_{3}\right.$-graded) Lie algebra, because the iterated $Z_{3}$-commutator $[A,[B, C]]$ cannot be expressed as a linear combination 
of two $Z_{3}$-commutators $[[A, B], C]$ and $[B,[A, C]]$ (i.e. the $Z_{3}$-graded analogue of the Jacobi identity does not exist here).

The derivations are naturally divided into three distinct classes, following their $Z_{3}$-grade. The derivations of $Z_{3}$-grade 1 and 2 are cubic nilpotent: if $\operatorname{grad}(A)=1$ or 2 ,

$$
\left(\operatorname{Der}_{A}\right)^{3} B=0 \text { for any } B
$$

for example, the simple calculus for $\mathrm{k}=1$ shows quite immediately that

$$
\begin{gathered}
D_{1}^{3} B:=\left[A,\left[A,[A, B]_{Z_{3}}\right]_{Z_{3}}\right]_{Z_{3}}=A^{3} B-j^{b} A^{2} B A-j^{b+1} A^{2} B A+j^{2 b+1} A B A^{2} \\
\quad-j^{b+2} A^{2} B A+j^{2 b+2} A B A^{2}+j^{2 b+3} A B A^{2}-j^{3 b+3} B A^{3}= \\
=A^{3} B-B A^{3}+j^{2 b}\left(j+j^{2}+j^{3}\right) B A^{2}-j^{b}\left(j+j^{2}+j^{3}\right) A^{2} B A=0
\end{gathered}
$$

where we have noted $\mathrm{b}:=\operatorname{grade}(\mathrm{B})$. The result comes from the fact that for any grade-1 matrix, the cube $A^{3}$ is proportional to the unit matrix and therefore commutes with any element $B \in \mathcal{A}$, and because the combination $j+j^{2}+j^{3}=$ $j+j^{2}+1$ is equal to 0 . The proof for the grade- 2 derivation is the same.

In contrast with what happens in the $Z_{2}$-graded Lie algebras, this derivation does not imply an analogue of the Jacobi identity, because it is not a derivation of the $Z_{3}$-graded commutator, i.e.

$$
\left[[X, Y]_{Z_{3}}, Z\right]_{Z_{3}}+\left[[Y, Z]_{Z_{3}}, X\right]_{Z_{3}}+\left[[Z, X]_{Z_{3}}, Y\right]_{Z_{3}} \neq 0
$$

An interesting example is provided by derivations of an associative algebra which is a ternary generalization of Grassmann algebra. Consider a free associative algebra with unit element, on which the following ternary relation is imposed:

$$
X Y Z=j Y Z X=j^{2} Z X Y
$$

Consider the simplest case with one generator only: then the whole algebra consists of three elements, $1, X$ and $X^{2}$, because $X^{3}=0$. If we want to define derivations satisfying the $Z_{3}$-graded Leibniz rule, i.e.

$$
\partial(X Y)=(\partial X) Y+j X(\partial Y)
$$

then it is easy to see that only three solutions are possible. They can be defined explicitly by their action on the three elements of our algebra:

$$
\begin{gathered}
\partial_{1}(X)=1 ; \quad \partial_{1}\left(X^{2}\right)=-j^{2} X ; \quad \partial_{1}(1)=0 ; \\
\partial_{2}(X)=X^{2} ; \quad \partial_{2}\left(X^{2}\right)=0 ; \quad \partial_{2}(1)=0 ; \\
\partial_{3}(X)=X ; \quad \partial_{3}\left(X^{2}\right)=-j^{2} X^{2} ; \quad \partial_{3}(1)=0
\end{gathered}
$$

The derivation $\partial_{1}$ is of grade $1, \partial_{2}$ is of grade 2 , whereas $\partial_{3}$ is of grade 0 . The two derivations $\partial_{1}$ and $\partial_{2}$ do not close under any binary relations, but they form a simple ternary algebra :

$$
\partial_{1} \partial_{2} \partial_{2}+\partial_{2} \partial_{1} \partial_{2}+\partial_{2} \partial_{2} \partial_{1}=-j^{2} \partial_{2}
$$




$$
\partial_{2} \partial_{1} \partial_{1}+\partial_{1} \partial_{2} \partial_{1}+\partial_{1} \partial_{1} \partial_{2}=-j^{2} \partial_{1} .
$$

We have already seen a similar non-associative ternary algebra realized in the set of 3-linear complex forms (Eq.19). Unfortunately, such $Z$-3-graded derivations cannot be realized on associative algebras satisfying the above permutation ternary rule with more than one independent generator; we can only repeat the construction by tensoring some number of identical realizations introduced above. The 1-dimensional version of this calculus has been worked out by W.S.Chung ([29])

The important fact is that the $Z_{3}$-graded derivations never close under a binary composition rule, but they can produce another derivation under a ternary composition rule.

In the associative algebra of $3 \times 3$ complex matrices we can also consider an exterior differential $d$ whose cube vanishes identically, $d^{3}=0$. Such a differential is defined as a $Z_{3}$ - graded commutator with a matrix from $\mathcal{A}_{1}$. In next ection we shall use such differentials to produce an extended version of usual gauge theories.

The associative $Z_{3}$-graded matrix algebra appears naturally as the algebra of linear transformations of another nilpotent graded associative algebra, which is a natural $Z_{3}$-graded generalisation of Grassmann algebras.

By analogy with the $Z_{2}$-graded Grassmann algebras spanned by the set of anti-commuting generators, we may introduce an associative algebra spanned by $N$ generators $\theta^{A}, A, B=1,2 \ldots N$, whose binary products $\theta^{A} \theta^{B}$ will be considered as $N^{2}$ independent quantities, whereas we shall impose a ternary analog of the anti-commutation relations:

$$
\theta^{A} \theta^{B} \theta^{C}=j \theta^{B} \theta^{C} \theta^{A}=j^{2} \theta^{C} \theta^{A} \theta^{B}
$$

A more precise formulation is to say that the algebra in question is the universal algebra defined by the above relations.

Corollary : The cube of any generator must vanish (because in this case the relation (1) amounts to $\left(\theta^{A}\right)^{3}=j\left(\theta^{A}\right)^{3}=0$; all the monomials of order 4 or higher are identically null (the proof that follows makes use of the associativity of the postulated product and of the relation 1): (the low braces are there just to indicate to which triple of $\theta$ 's the circular permutation is being applied)

$$
\underbrace{\theta^{A} \theta^{B} \theta^{C}} \theta^{D}=j \theta^{B} \underbrace{\theta^{C} \theta^{A} \theta^{D}}=j^{2} \underbrace{\theta^{B} \theta^{A} \theta^{D}} \theta^{C}=\theta^{A} \underbrace{\theta^{D} \theta^{B} \theta^{C}}=j \theta^{A} \theta^{B} \theta^{C} \theta^{D} ;
$$

therefore, as $1-j \neq 0$, one has $\theta^{A} \theta^{B} \theta^{C} \theta^{D}=0$.

The dimension of this $Z_{3}$-graded generalization of Grassmann algebra is equal to $N+N^{2}+\left(N^{3}-N\right) / 3$; we may also add a "neutral" element denoted by 1 and commuting with all other generators.

One can note a dissymmetry between the components of this algebra with the grades 1 et 2 : as a matter of fact, there are $N$ elements of grade 1 (the $\theta$ 's) and $N^{2}$ elements of grade $2(\theta \theta)$. 
A natural way to re-establish the symmetry is to introduce the set of $N$ "conjugate" generators, $\bar{\theta}^{A}$, of grade 2 , that satisfy conjugate ternary relations (in which $j$ is replaced by $j^{2}$ ):

$$
\bar{\theta}^{A} \bar{\theta}^{B} \bar{\theta}^{C}=j^{2} \bar{\theta}^{B} \bar{\theta}^{C} \bar{\theta}^{A}
$$

The ternary relation between the $\theta^{A}$ 's can be interpreted as follows: $\theta^{A} \underbrace{\theta^{B} \theta}=j \underbrace{\theta^{B} \theta^{C}} \theta^{A}$, which suggests the following relations between the generators $\theta^{A}$ and $\bar{\theta}^{B}$ :

$$
\theta^{A} \bar{\theta}^{B}=j \bar{\theta}^{B} \theta^{A}, \quad \bar{\theta}^{B} \theta^{A}=j^{2} \theta^{A} \bar{\theta}^{B} .
$$

The $Z_{3}$-graded algebra so defined can be naturally divided in three parts, of grade 0,1 et 2 respectively, with the dimensions of the sub-spaces of grades 1 and 2 being equal: one can write symbolically $A=A_{0}+A_{1}+A_{2}$, where

$A_{0}$ contains: 1, $\theta^{A} \bar{\theta}^{B}, \theta^{A} \theta^{B} \theta^{C}, \bar{\theta}^{A} \bar{\theta}^{B} \bar{\theta}^{C}, \theta^{A} \theta^{B} \bar{\theta}^{C} \bar{\theta}^{D}$ and $\theta^{A} \theta^{B} \theta^{C} \bar{\theta}^{D} \bar{\theta}^{E} \bar{\theta}^{F}$;

$A_{1}$ contains: $\theta^{A}, \bar{\theta}^{B} \bar{\theta}^{C}, \theta^{A} \theta^{B} \bar{\theta}^{C}, \theta^{A} \bar{\theta}^{A} \bar{\theta}^{B} \bar{\theta}^{C}$,

and $A_{2}$ contains: $\bar{\theta}^{A}, \theta^{A} \theta^{B}, \theta^{A} \bar{\theta}^{B} \bar{\theta}^{C}, \theta^{A} \theta^{B} \theta^{C} \bar{\theta}^{D}$.

In the case of usual $Z_{2}$-graded Grassmann algebras the anti-commutation between the generators of the algebra and the assumed associativity imply automatically the fact that all grade 0 elements commute with the rest of the algebra, while any two elements of grade 1 anti-commute.

In the case of the $Z_{3}$-graded generalization such an extension of ternary and binary relations does not follow automatically, and must be imposed explicitly. If we decide to extend these relations to all elements of the algebra having a well-defined grade (i.e. the monomials in $\theta$ 's and $\bar{\theta}$ 's, then many additional expressions must vanish, e.g.:

$$
\theta^{A} \underbrace{\theta^{B} \bar{\theta}^{C}}=\underbrace{\theta^{B} \bar{\theta}^{C}} \theta^{A}=\theta^{B} \underbrace{\bar{\theta}^{C} \theta^{A}}=\bar{\theta}^{C} \theta^{A} \theta^{B}=0 ;
$$

because on the one side, $\theta^{A} \bar{\theta}^{C}$ is of grade 0 and commutes with all other elements; at the same time, commuting $\bar{\theta}^{C}$ with $\theta^{A} \theta^{B}$ one gets twice the factor $j^{2}$, which leads to the overall factor $j \bar{\theta}^{C} \theta^{A} \theta^{B}$; this produces a contradiction which can be solved only by supposing that $\theta^{A} \theta^{B} \bar{\theta}^{C}=0$. The resulting $Z_{3}$-graded algebra contains only the following products of generators:

$$
A_{1}=\theta,\{\bar{\theta} \bar{\theta}\} ; \quad A_{2}=\bar{\theta}, \quad\{\theta \theta\} ; \quad A_{0}=\{\theta \bar{\theta}\}, \quad\{\theta \theta \theta\}, \quad\{\bar{\theta} \bar{\theta} \bar{\theta}\}
$$

Let us note that the set of grade 0 (which obviously forms a sub-algebra of the $Z_{3}$-graded Grassmann algebra) contains the products which could symbolize the only observable combinations of quark fields in quantum chromodynamics based on $S U(3)$-symmetry.

If we reorder the basis of our algebra, with all the elements of grade 0 first, next all the elements of grade 1 and finally the elements of grade 2 in a onecolumn vector, a general linear transformation that would leave these entries 
in the same order can be symbolized by a matrix whose entries have a definite $Z_{3}$-grade placed as follows:

$$
\left(\begin{array}{lll}
0 & 2 & 1 \\
1 & 0 & 2 \\
2 & 1 & 0
\end{array}\right)\left(\begin{array}{l}
0 \\
1 \\
2
\end{array}\right)=\left(\begin{array}{l}
0 \\
1 \\
2
\end{array}\right)
$$

Under the action of such a matrix, the position of the three grades does not change in the resulting column; we shall call such an operator a grade 0 matrix. We can introduce two other kinds of matrices that raise all the grades by 1 (resp. by 2), and call them respectively grade 1 and grade 2 matrices:

$$
\left(\begin{array}{lll}
1 & 0 & 2 \\
2 & 1 & 0 \\
0 & 2 & 1
\end{array}\right)\left(\begin{array}{l}
0 \\
1 \\
2
\end{array}\right)=\left(\begin{array}{l}
1 \\
2 \\
0
\end{array}\right), \text { and }\left(\begin{array}{lll}
2 & 1 & 0 \\
0 & 2 & 1 \\
1 & 0 & 2
\end{array}\right)\left(\begin{array}{l}
0 \\
1 \\
2
\end{array}\right)=\left(\begin{array}{l}
2 \\
0 \\
1
\end{array}\right)
$$

(the numbers $0,1,2$ symbolize the grades of the respective entries in the matrices). The notions of hypertrace and hyperdeterminant generalizing the corresponding notions of supertrace and superdeterminant been successfully introduced and investigated recently by B. Le Roy ([31). If we restrict the character of the matrices, admitting only complex-valued matrix elements, then the grades 0,1 and 2 will reduce themselves to the following three types of $3 \times 3$-block matrices:

$$
\left(\begin{array}{lll}
a & 0 & 0 \\
0 & b & 0 \\
0 & 0 & c
\end{array}\right), \quad\left(\begin{array}{lll}
0 & \alpha & 0 \\
0 & 0 & \beta \\
\gamma & 0 & 0
\end{array}\right), \quad\left(\begin{array}{lll}
0 & 0 & \gamma \\
\alpha & 0 & 0 \\
0 & \beta & 0
\end{array}\right)
$$

representing arbitrary matrices with respective grade 0,1 and 2 , i.e. the $Z_{3^{-}}$ graded algebra introduced in the beginning of this Section.

Let $\eta$ be a matrix of grade 1 ; we can define a formal "differential" on the $Z_{3}$-graded algebra of 3 matrices as follows:

$$
d B:=[\eta, B]_{Z_{3}}=\eta B-j^{b} B \eta
$$

It is easy to show that $d(B C)=(d B) C+j^{b} B(d C)$ and that $d^{3}=0$. The first identity is trivial, whereas the last one follows from the fact that $\eta^{3}=I d$ does commute with all the elements of the algebra.

It is also easy to check that $\operatorname{Im}(d) \subseteq \operatorname{Ker}\left(d^{2}\right)$, and $\operatorname{Im}\left(d^{2}\right) \subseteq \operatorname{Ker}(d)$

\section{Matrix realization of a $Z_{3}$-graded gauge theory}

Let $\mathcal{A}$ be an associative algebra with unit element, and let $\mathcal{H}$ be a free left module over this algebra. Let $A$ be an $\mathcal{A}$-valued 1 -form defined on a differential manifold $M$, and let $\Phi$ be a function on the manifold $M$ with values in the module $\mathcal{H}$. We shall introduce the covariant differential as usual:

$$
D \Phi:=d \Phi+A \Phi
$$


If the module is a free one, any of its elements $\Phi$ can be represented by an appropriate element of the algebra acting on a fixed element of $\mathcal{H}$, so that one can always write $\Phi=B \Phi_{o}$; then the action of the group of automorphisms of $\mathcal{H}$ can be translated as the action of the same group on the algebra $\mathcal{A}$.

Let $U$ be a function defined on $M$ with its values in the group of the automorphisms of $\mathcal{H}$. The definition of a covariant differential is equivalent with to the requirement $D U^{-1} B=U^{-1} D B$; as in the usual case, this leads to the following well-known transformation for the connection 1-form $A$ :

$$
A \Rightarrow U^{-1} A U+U^{-1} d U
$$

But here, unlike in the usual theory, the second covariant differential $D^{2} \Phi$ is not an automorphism: as a matter of fact, we have:

$$
D^{2} \Phi=d(d \Phi+A \Phi)+A(d \Phi+A \Phi)=d^{2} \Phi+d A \Phi+j A d \Phi+A d \Phi+A^{2} \Phi
$$

the expression containing $d^{2} \Phi$ and $d \Phi$, whereas $D^{3} \Phi$ is an automorphism, because it contains only $\Phi$ multiplied on the left by an algebra-valued 3 -form:

$$
\begin{gathered}
D^{3} \Phi=d\left(D^{2} \Phi\right)+A\left(D^{2} \Phi\right), \text { which gives explicitly : } \\
d\left(d^{2} \Phi+d A \Phi+j A d \Phi+A^{2} \Phi\right)+A\left(d^{2} \Phi+d A \Phi+j A d \Phi+A d \Phi+A^{2} \Phi\right)
\end{gathered}
$$

With a direct calculus one observes that all the terms containing $d \Phi$ or $d^{2} \Phi$ simplify because of the identity $1+j+j^{2}=0$, leaving only

$$
D^{3} \Phi=\left(d^{2} A+d\left(A^{2}\right)+A d A+A^{3}\right) \Phi=\left(D^{2} A\right) \Phi:=\Omega \quad \Phi ;
$$

Obviously, because $D\left(U^{-1} \Phi\right)=U^{-1}(D \Phi)$, one also has:

$$
D^{3}\left(U^{-1} \Phi\right)=U^{-1}\left(D^{3} \Phi\right)=U^{-1} \Omega \Phi=U^{-1} \Omega U U^{-1} \Phi,
$$

which proves that the 3 -form $\Omega$ transforms as usual, $\Omega \Rightarrow U^{-1} \Omega U$ when the connection 1-form transforms according to the law: $A \Rightarrow U^{-1} A U+U^{-1} d U$.

It can be also proved by a direct calculus that the curvature 3 -form $\Omega$ does vanish identically for $A=U^{-1} d U$. This computation illustrates very well the technique of the $Z_{3}$-graded exterior differential calculus introduced above: as a matter of fact, one has

$$
d\left(U^{-1} d U\right)=d U^{-1} d U+U^{-1} d^{2} U
$$

so that the term corresponding to $d^{2} A$ gives:

$$
d^{2}\left(U^{-1} d U\right)=d^{2} U^{-1} d U+j d U^{-1} d^{2} U+d U^{-1} d^{2} U
$$

next, the term corresponding to $d\left(A^{2}\right)=d\left(U^{-1} d U U^{-1} d U\right)$ gives

$$
d U^{-1} d U U^{-1} d U+U^{-1} d^{2} U U^{-1} d U+j U^{-1} d U d U^{-1} d U+j U^{-1} d U U^{-1} d^{2} U
$$




$$
\text { whereas } A d A=U^{-1} d U d U^{-1} d U+U^{-1} d U U^{-1} d^{2} U
$$

finally, the term $A^{3}=U^{-1} d U U^{-1} d U U^{-1} d U$ can be written as $-d U^{-1} d U U^{-1} d U$ by virtue of the identity $d U U^{-1}=-U d U^{-1}$ which follows from the Leibniz rule applied to $U U^{-1}=I d$, i.e. $d\left(U U^{-1}\right)=d U U^{-1}+U d U^{-1}=0$. Using this identity whenever possible, and replacing $1+j$ by $-j^{2}$, we can reduce the whole expression to the following sum of three terms

$$
d^{2} U^{-1} d U+U^{-1} d^{2} U U^{-1} d U-j^{2} U^{-1} d U d U^{-1} d U
$$

whose vanishing does not at all seem obvious. However, it is not very difficult to prove that this expression is identically null. First of all, it is enough to prove the vanishing of the expression

$$
d^{2} U^{-1}+U^{-1} d^{2} U U^{-1}-j^{2} U^{-1} d U d U^{-1},
$$

because all the three terms contain the same factor $d U$ on the right; then, by multiplying on the left by $U$, we get $U d^{2} U^{-1}+d^{2} U U^{-1}-j^{2} d U d U^{-1}$

At this point let us note that $d^{2}\left(U U^{-1}\right)=d^{2}(I d)=0$, but then, according to our $Z_{3}$-graded Leibniz rule,

$$
\begin{gathered}
d^{2}\left(U U^{-1}\right)=d\left(d U U^{-1}+U d U^{-1}\right)=d^{2} U U^{-1}+j d U d U^{-1}+d U d U^{-1}+U d^{2} U^{-1} \\
\text { so that } U d^{2} U^{-1}+d^{2} U U^{-1}=-d U d U^{-1}-j d U d U^{-1}, \text { therefore } \\
-d U d U^{-1}-j d U d U^{-1}-j^{2} d U d U^{-1}=\left(1+j+j^{2}\right) d U d U^{-1}=0
\end{gathered}
$$

It is amusing to look at one of the simplest possible realizations of this model in the $Z_{3}$-graded algebra of $3 \times 3$ complex matrices. In this case the matrices represent both functions and forms, so that it is easy to perform the calculus of the 3 -form $\Omega$ and in particular to determine the condition on the connection 1-form $A$ leading to $\Omega=0$, i.e. defining the set of connections $A$ that are pure gauges.

$$
\text { Let } A=\left(\begin{array}{ccc}
0 & \alpha & 0 \\
0 & 0 & \beta \\
\gamma & 0 & 0
\end{array}\right) \text { and } \eta=\left(\begin{array}{ccc}
0 & 1 & 0 \\
0 & 0 & 1 \\
1 & 0 & 0
\end{array}\right) \text { Then the condition } \Omega=0 \text { leads }
$$
to the following equation for the coefficients $\alpha, \beta, \gamma$ :

$$
(\alpha+\beta+\gamma)+\alpha \beta+\beta \gamma+\gamma \alpha+\alpha \beta \gamma=0,
$$

or in a more symmetric form, $(\alpha+1)(\beta+1)(\gamma+1)=1$

The independent solutions displaying full symmetry under the action of the permutation group $S_{3}$ are found by putting

$$
\alpha+1=\beta+1=\gamma+1=1 \text { or } j \text { or } j^{2} \text {; }
$$

Another set of solutions is obtained with

$$
\alpha+1=1, \quad \beta+1=j, \quad \gamma+1=j^{2}
$$

and by performing all possible permutations of $\alpha, \beta$ and $\gamma$. The analogue of a gauge transformation acting on the connection form $A$ is given by a usual formula,

$$
A \rightarrow A^{\prime}=U^{-1} A U+U^{-1} d U
$$


Then it is easy to show that for any $U \in \mathcal{A}$ one has $\Omega^{\prime}=U^{-1} \Omega U$, but the covariant derivative $D$ transforms properly only if $U \in$ cal $A_{0}$; in other case, one has $U^{-1}(d+A) U=d+j^{u} A$. Let $U \in \mathcal{A}_{0}$. Then,

$$
\text { if } U=\left(\begin{array}{ccc}
x & 0 & 0 \\
0 & y & 0 \\
0 & 0 & z
\end{array}\right) \text {, one has } U^{-1} d U=\left(\begin{array}{ccc}
0 & 0 & \left(\frac{z}{x}-1\right) \\
\left(\frac{x}{y}\right)-1 & 0 & 0 \\
0 & \left(\frac{y}{z}\right)-1 & 0
\end{array}\right)
$$

which obviously satisfies the equation (52), therefore corresponding to the vanishing curvature 3 -form $\Omega$. Indeed,

$$
\left(\left(\frac{z}{x}-1\right)+1\right)\left(\left(\frac{x}{y}-1\right)+1\right)\left(\left(\frac{y}{z}-1\right)+1\right)=\frac{z}{x} \frac{x}{y} \frac{y}{z}=1
$$

\section{6. $Z_{3}$-graded Exterior Differential Calculus}

The associative algebra of functions we shall be dealing with is generated by $N$ generators $\xi^{k}$, which need not to commute. We shall assume more general binary relations of the type

$$
\xi^{i} \xi^{k}=\xi^{k} \xi^{i}+\epsilon^{i k}=\xi^{i} \xi^{k}+\epsilon^{k i}+\epsilon^{i k},
$$

so that obviously one must have $\epsilon^{i k}=-\epsilon^{k i}$; the variables $\xi^{k}$ span a Heisenberg algebra in an even dimensional case.

We postulate that the partial derivatives satisfy the usual Leibniz rule:

$$
\partial_{i} \xi^{k}=\delta_{i}^{k} ; \quad \partial_{i}\left(\xi^{k} \xi^{l}\right)=\delta_{i}^{k} \xi^{l}+\xi^{k} \delta_{i}^{l}, \quad \text { and } \quad \partial_{i}\left(\epsilon^{k m}\right)=0,
$$

and that it holds for a product of any two functions of the variables $\xi^{k}$. Our algebra being associative, we have also $\partial_{i} \partial_{k}=\partial_{k} \partial_{i}$. Let the definition of the differential $d f$ of a function $f$ coincide with the usual one:

$$
d f=\frac{\partial f}{\partial \xi^{k}} d \xi^{k}
$$

When formally computing higher-order differentials, we shall suppose that our exterior differential operator $d$ obeys the generalized graded Leibniz rule:

$$
d(\omega \phi)=d \omega \phi+j^{\text {grade }(\omega)} \omega d \phi
$$

and that the grades add modulo 3 under the associative multiplication of exterior forms; the functions are of grade 0 , and the operator $d$ raises the grade of any form by 1 , which means that $d \xi^{k}$ is a 1 -form whose $Z_{3}$-grade is 1 by definition; when applied two times, by iteration, $d$ will produce a new entity, which we shall call a 1-form of grade 2 , denoted by $d^{2} \xi^{k}$. Finally, we require that $d^{3}=0$.

We shall suppose that the $Z_{3}$-graded algebra generated by the forms $d \xi^{i}$ and $d^{2} \xi^{k}$ behaves as a left module over the algebra of smooth functions of $\xi^{\prime}$ s. In 
other words, we shall be able to multiply the forms $d \xi^{i}, d^{2} \xi^{k}, d \xi^{i} d \xi^{k}$, etc. by the functions on the left only; right multiplication will just not be considered here. That is why we will write by definition, e.g.

$$
d\left(\xi^{i} \xi^{k}\right):=\xi^{i} d \xi^{k}+\xi^{k} d \xi^{i}
$$

Let us note that in contrast to the $Z_{2}$-graded case, the forms are treated as a whole, even when multiplied from the left by an arbitrary function; that means that we cannot identify e.g.

$$
\left(\omega_{i} d \xi^{i}\right)\left(\phi_{k} d \xi^{k}\right) \quad \text { with } \quad\left(\omega_{i} \phi_{k}\right) d \xi^{i} d \xi^{k}
$$

It is equivalent to say that the products of functions by forms are to be understood in the sense of tensor products, which is associative, but noncommutative.

Nevertheless, such an identification can be done for the forms of maximal degree (i.e. 3), which contain the products of the type $d \xi^{i} d \xi^{k} d \xi^{m}$ or $d \xi^{i} d^{2} \xi^{m}$, whose exterior differentials vanish irrespective of the order of the multiplication; as a matter of fact,

$$
\left.d\left(\left(\alpha_{i} d \xi^{i}\right)\left(\beta_{k} d \xi^{k}\right)\left(\gamma_{m} d \xi^{m}\right)\right)=d\left(\left(\alpha_{i} \beta_{k} \gamma_{m}\right) d \xi^{i} d \xi^{k} d \xi^{m}\right)\right)=0 .
$$

With the $Z_{3}$-graded Leibniz rule so established, the postulate $d^{3}=0$ suggests the ternary and binary commutation rules for the differentials $d \xi^{i}$ and $d^{2} \xi^{k}$. To begin with, consider the differentials of a function of the coordinates $\xi^{k}$, where the "first differential" $d f$ coincides with the usual one:

$$
\begin{gathered}
d f:=\left(\partial_{i} f\right) d \xi^{i} ; \quad d^{2} f:=\left(\partial_{k} \partial_{i} f\right) d \xi^{k} d \xi^{i}+\left(\partial_{i} f\right) d^{2} \xi^{i} ; \\
d^{3} f=\left(\partial_{m} \partial_{k} \partial_{i} f\right) d \xi^{m} d \xi^{k} d \xi^{i}+\left(\partial_{k} \partial_{i} f\right)\left[d^{2} \xi^{k} d \xi^{i}+j d \xi^{i} d^{2} \xi^{k}+d \xi^{k} d^{2} \xi^{i}\right]
\end{gathered}
$$

(we recall that the last part of the differential, $\left(\partial_{i} f\right) d^{3} \xi^{i}$, vanishes by virtue of the postulate $\left.d^{3} \xi^{i}=0\right)$.

Supposing that partial derivatives commute, exchanging the summation indices $i$ et $k$ in the last expression and replacing $1+j$ by $-j^{2}$, we arrive at the following two conditions that lead to the vanishing of $d^{3} f$ :

$$
d \xi^{m} d \xi^{k} d \xi^{i}+d \xi^{k} d \xi^{i} d \xi^{m}+d \xi^{i} d \xi^{m} d \xi^{k}=0 \quad ; \quad d^{2} \xi^{k} d \xi^{i}-j^{2} d \xi^{i} d^{2} \xi^{k}=0
$$

which lead in turn to the following relations:

$$
d \xi^{i} d \xi^{k} d \xi^{m}=j d \xi^{k} d \xi^{m} d \xi^{i}, \text { and } d \xi^{i} d^{2} \xi^{k}=j d^{2} \xi^{k} d \xi^{i}
$$

By extending these rules to all the expressions with a well-defined grade, and applying the associativity of the $Z_{3}$-exterior product, we see that all the expressions of the type $d \xi^{i} d \xi^{k} d \xi^{m} d \xi^{n}$ and $d \xi^{i} d \xi^{k} d^{2} \xi^{m}$ must vanish, and along with them, also the monomials of higher order that would contain them as 
factors. Still, this is not sufficient in order to satisfy the rule $d^{3}=0$ on all the forms spanned by the generators $d \xi^{1}$ and $d^{2} \xi^{k}$. It can be proved easily that the expressions containing $d^{2} \xi^{i} d^{2} \xi^{k}$ must vanish, too. For example, if we take the particular 1-form $\xi^{i} d \xi^{k}$ and apply to it the operator $d$, we get

$$
\begin{gathered}
d\left(\xi^{i} d \xi^{k}\right)=d \xi^{i} d \xi^{k}+\xi^{i} d^{2} \xi^{k} \\
d^{2}\left(\xi^{i} d \xi^{k}\right)=d^{2} \xi^{i} d \xi^{k}+(1+j) d \xi^{i} d^{2} \xi^{k}=d^{2} \xi^{i} d \xi^{k}-d^{2} \xi^{k} d \xi^{i}
\end{gathered}
$$

which leads to $d^{3}\left(\xi^{i} d \xi^{k}\right)=d^{2} \xi^{i} d^{2} \xi^{k}-d^{2} \xi^{k} d^{2} \xi^{i}$; then, if we want to keep both the associativity of the "exterior product" and the ternary rule for the entities of grade 2, i.e. $d^{2} \xi^{i} d^{2} \xi^{k} d^{2} \xi^{m}=j^{2} d^{2} \xi^{k} d^{2} \xi^{m} d^{2} \xi^{i}$, then the only solution is to impose $d^{2} \xi^{i} d^{2} \xi^{k}=0$ and to set forward the additional rule declaring that any expression containing four or more operators $d$ must identically vanish.

With these rules set, we can check that $d^{3}=0$ on all the forms, whatever their grade or degree. Let us show how such a calculus works on the example of a 1 -form $\omega=\omega_{k} d \xi^{k}$ :

$$
\begin{gathered}
d\left(\omega_{k} d \xi^{k}\right)=\left(\partial_{i} \omega_{k}\right) d \xi^{i} d \xi^{k}+\omega_{k} d^{2} \xi^{k} \\
d^{2}\left(\omega_{k} d \xi^{k}\right)=\left(\partial_{m} \partial_{i} \omega_{k}\right) d \xi^{m} d \xi^{i} d \xi^{k}+\left(\partial_{i} \omega_{k}\right)\left(d^{2} \xi^{i} d \xi^{k}+j d \xi^{i} d^{2} \xi^{k}\right)+\partial_{i} \omega_{k} d \xi^{i} d^{2} \xi^{k}
\end{gathered}
$$

exchanging the summation indices $i$ and $k$ in two last terms, using $j+1=-j^{2}$ and the commutation relations between $d \xi^{k}$ and $d^{2} \xi^{i}$, we can write

$$
d^{2}\left(\omega_{k} d \xi^{k}\right)=\left(\partial_{m} \partial_{i} \omega_{k}\right) d \xi^{m} d \xi^{i} d \xi^{k}+\left(\partial_{i} \omega_{k}-\partial_{k} \omega_{i}\right) d^{2} \xi^{i} d \xi^{k}
$$

where it is interesting to note how the usual anti-symmetric exterior differential appears as a part of the whole expression.

The natural symmetry between $j$ et $j^{2}$ which leads to the possibility of choosing one of these two complex numbers as the generator of the group $Z_{3}$, and simultaneous interchanging the rôles between the grades 1 and 2 suggests that we could extend the notion of complex conjugation $j \Rightarrow(j)^{*}=j^{2}$, with $\left((j)^{*}\right)^{*}=j$, to the algebra of $Z_{3}$-graded exterior forms and the operator $d$ itself.

It does not seem reasonable to use the "second differentials" $d^{2} x^{i}$ as the objects conjugate to the "first differentials" $d x^{i}$, because the rules of $Z_{3}$-graded exterior differentiation we have imposed break the symmetry between these two kinds of differentials: remember that the products $d x^{i} d x^{k}$, and $d x^{i} d x^{k} d x^{m}$ are admitted, while we require that $d^{2} x^{i} d^{2} x^{k}$ and $d^{2} x^{i} d^{2} x^{k} d^{2} x^{m}$ must vanish.

This suggests the introduction of a "conjugate" differential $\delta$ of grade 2, the image of the differential $d$ under the conjugation $*$, satisfying the following conjugate relations:

$$
\delta x^{i} \delta x^{k} \delta x^{m}=j^{2} \delta x^{k} \delta x^{m} \delta x^{i}, \quad \delta x^{i} \delta^{2} x^{k}=j^{2} \delta^{2} x^{k} \delta x^{i} .
$$

One notes that $\delta^{2} x^{k}$ is of grade $1(2+2=4=1(\bmod 3))$.

All the relations existing between the operator $d$ and the exterior forms generated by $d x^{i}$ and $d^{2} x^{k}$ are faithfully reproduced under the conjugation $*$ if we 
consider the $Z_{3}$-graded algebra generated by the entities $\delta x^{i}$ and $\delta^{2} x^{k}$ as a right module over the algebra of functions $F(M)$, with the operator $\delta$ acting on the right on this module.

The rules $d^{3}=0$ and $\delta^{3}=0$ suggest their natural extension: $d \delta=\delta d=0$

To conclude, we must stress the following point: although algebraically consistent, the $Z_{3}$-graded exterior differential calculus does not yet have any deep geometrical meaning, unlike the case of usual $Z_{2}$-grading, with the duality between the exterior p-forms and p-cells and complexes,integration, and GreenStokes formulae. In our case, for example, we would like to be able to extend these formulae as follows:

$$
\int_{C} d^{2} \omega=\int_{\partial C} d \omega=\int_{\partial^{2} C} \omega
$$

where the operation $\partial$, when applied to a "complex" $C$, gives a new complex $\partial C$ of lower dimension, but with the possibility of applying it twice, and with $\partial^{3} C=0$ for any $C$.

\section{7. $Z_{3}$-graded Gauge Theory on Principal and Linear Bundles}

The curvature 3-form $\Omega=d^{2} A+d\left(A^{2}\right)+A d A+A^{3}$ is of grade 0 ; therefore it must be decomposed along the elements $d x^{i} d x^{k} d x^{m}$ and $d^{2} x^{i} d x^{k}$. Here is how we can compute its components in a local coordinate system. By definition, $A=A_{i} d x^{i}$, so we have:

$$
\begin{gathered}
d A=\partial_{i} A_{k} d x^{i} d x^{k}+A_{k} d^{2} x^{k} \\
d^{2} A=\partial_{m} \partial_{i} A_{k} d x^{m} d x^{i} d x^{k}+\partial_{i} A_{k} d^{2} x^{i} d x^{k}+j \partial_{i} A_{k} d x^{i} d^{2} x^{k}+\partial_{i} A_{k} d x^{i} d^{2} x^{k}
\end{gathered}
$$

Replacing $1+j$ by $-j^{2}$, and taking into account $d x^{k} d^{2} x^{i}=j d^{2} x^{i} d x^{k}$, we get:

$$
d^{2} A=\left(\partial_{m} \partial_{i} A_{k}\right) d x^{m} d x^{i} d x^{k}+\left(\partial_{i} A_{k}-\partial_{k} A_{i}\right) d^{2} x^{i} d x^{k} ;
$$

Then, $d\left(A^{2}\right)+A d A=d A A+j A d A+A d A=d A A-j^{2} A d A$, which leads to

$\left(\partial_{i} A_{k} A_{m}\right) d x^{i} d x^{k} d x^{m}-j^{2}\left(A_{m} \partial_{i} A_{k}\right) d x^{m} d x^{i} d x^{k}+A_{k} A_{m} d^{2} x^{k} d x^{m}-j^{2} A_{m} A_{k} d x^{m} d^{2} x^{k}$ and due to the relations $d x^{m} d^{2} x^{k}=j d^{2} x^{k} d x^{m}$ et $d x^{m} d x^{i} d x^{k}=j d x^{i} d x^{k} d x^{m}$,

$$
d\left(A^{2}\right)+A d A=\left(A_{m} \partial_{i} A_{k}-\partial_{i} A_{k} A_{m}\right) d x^{m} d x^{i} d x^{k}+\left(A_{k} A_{m}-A_{m} A_{k}\right) d^{2} x^{k} d x^{m} .
$$

Finally, as $A^{3}=A_{i} A_{k} A_{m} d x^{i} d x^{k} d x^{m}$, the curvature 3 -form can be written in local coordinates as follows:

$$
\begin{gathered}
\Omega=d^{2} A+d\left(A^{2}\right)+A d A+A^{3}=\Omega_{i k m} d x^{i} d x^{k} d x^{m}+F_{i k} d^{2} x^{i} d x^{k} \\
\text { where } \Omega_{i k m}:=\partial_{i} \partial_{k} A_{m}+A_{i} \partial_{k} A_{m}-\partial_{k} A_{m} A_{i}+A_{i} A_{k} A_{m}, \\
\text { and } F_{i k}:=\partial_{i} A_{k}-\partial_{k} A_{i}+A_{i} A_{k}-A_{k} A_{i} ;
\end{gathered}
$$

In $F_{i k}$ we easily recognize the 2-form of curvature of the usual gauge theories.

We know that the expression $F_{i k}$ is covariant with respect to the gauge 
transformations; on the other hand, the 3-form $\Omega$ is also covariant; therefore, the local expression $\Omega_{i j k}$ must be covariant, too. As a matter of fact, it can be expressed as a combination of covariant derivatives of the 2 -form $F_{i k}$.

In order to find the covariant expression of $\Omega_{i k m}$, it suffices to recall that due to the particular symmetry of the ternary exterior product $d x^{i} d x^{k} d x^{m}$, we can replace $\Omega_{i k m}$ by $\frac{1}{3}\left(\Omega_{i k m}+j^{2} \Omega_{k m i}+j \Omega_{m i k}\right)$ and analyze the abelian case, when this expression reduces itself to $\Omega_{i k m}=\partial_{i} \partial_{k} A_{m}$. Substituting for $\partial_{i} \partial_{k} A_{m}$ the equivalent expression $\frac{1}{3}\left(\partial_{i} \partial_{k} A_{m}+j^{2} \partial_{k} \partial_{m} A_{i}+j \partial_{m} \partial_{i} A_{k}\right)$ and writing it as $\frac{1}{3}\left(j\left(\partial_{k} \partial_{m} A_{i}-\partial_{i} \partial_{k} A_{m}\right)+j\left(\partial_{m} \partial_{i} A_{k}-\partial_{i} \partial_{k} A_{m}\right)\right)$, because $1=-j-j^{2}$,

we can easily recognize $\frac{1}{3}\left(j \partial_{i}\left[\partial_{m} A_{k}-\partial_{k} A_{m}\right]+j^{2} \partial_{k}\left[\partial_{m} A_{i}-\partial_{i} A_{k}\right]\right)$;

which in a general non-abelian case must lead to the following expression:

$$
\Omega_{i k m}=\frac{1}{3}\left[j D_{i} F_{m k}+j^{2} D_{k} F_{m i}\right]
$$

or equivalently $\Omega_{i k m}=-\frac{1}{6}\left[D_{i} F_{m k}+D_{k} F_{m i}\right]+\frac{i \sqrt{3}}{6}\left[D_{i} F_{m k}-D_{k} F_{m i}\right]$

A similar construction in the case of linear connection and curvature is also easy to perform. Let $\left\{\mathbf{e}_{k}\right\}$ denote the set of $N$ independent vectors defined at any point of our space (which we suppose locally isomorphic to $R^{N}$ ), forming a basis. We define the covariant differential of $\mathbf{e}_{k}$ by means of the covariant derivatives of the $\mathbf{e}_{k}$ which define the connection coefficients $\Gamma_{i k}^{l}$ :

$$
\nabla \mathbf{e}_{k}=\nabla_{i} \mathbf{e}_{k} d \xi^{k}=\Gamma_{i k}^{l} \mathbf{e}_{l} d \xi^{i}
$$

Now, when applying this operation second time, we get:

$$
\nabla^{2} \mathbf{e}_{k}=\partial_{m} \Gamma_{i k}^{l} \mathbf{e}_{l} d \xi^{m} d \xi^{i}+\Gamma_{i k}^{l}\left(\nabla_{m} \mathbf{e}_{k}\right) d \xi^{m} d \xi^{i}+\Gamma_{i k}^{l} \mathbf{e}_{l} d^{2} \xi^{i}
$$

which in view of the definition of $\nabla_{m} \mathbf{e}_{k}$ can be written as:

$$
\nabla^{2} \mathbf{e}_{k}=\left(\partial_{m} \Gamma_{i k}^{l}+\Gamma_{m j}^{l} \Gamma_{i k}^{j}\right) \mathbf{e}_{l} d \xi^{m} d \xi^{i}+\Gamma_{i k}^{l} \mathbf{e}_{l} d^{2} \xi^{i}
$$

In the usual differential geometry we would set by definition $d^{2} \xi^{k}=0$, and $d \xi^{i} d \xi^{k}=-d \xi^{k} d \xi^{i}$, which automatically leads to the well-known expression

$$
\nabla^{2} \mathbf{e}_{k}=R_{m i k}^{l} \mathbf{e}_{l} d \xi^{m} \wedge d \xi^{m}
$$

$$
\begin{gathered}
\text { with } R_{m i k}^{l}=\partial_{m} \Gamma_{i k}^{l}-\partial_{i} \Gamma_{m k}^{l}+\Gamma_{m j}^{l} \Gamma_{i k}^{j}-\Gamma_{i j}^{l} \Gamma_{m k}^{j}, \\
\text { and } d \xi^{m} \wedge d \xi^{i}=\frac{1}{2}\left(d \xi^{m} \otimes d \xi^{i}-d \xi^{i} \otimes d \xi^{m}\right)
\end{gathered}
$$

Here we no longer assume $d^{2} \xi^{k}=0$ anymore, nor any particular symmetry of the tensorial product of the differenitals $d \xi^{k} \otimes d \xi^{m}$. Therefore we must write instead:

$$
\nabla^{2} \mathbf{e}_{k}=\left(\frac{1}{2} R_{m i k}^{l}+\frac{1}{2} P_{m i k}^{l}\right) \mathbf{e}_{l} d \xi^{m} d \xi^{i}+\Gamma_{i k}^{l} \mathbf{e}_{l} d^{2} \xi^{i}
$$


with a new entity

$$
P_{m i k}^{l}=\partial_{m} \Gamma_{i k}^{l}+\partial_{i} \Gamma_{m k}^{l}+\Gamma_{m j}^{l} \Gamma_{i k}^{j}+\Gamma_{i j}^{l} \Gamma_{m k}^{j}
$$

Note that $P_{m i k}^{l}$ does not transform as a tensor under a change of coordinates, but instead obeys a non-homogeneous transformation law, like the connection coefficients. Now, if we calculate the third covariant derivative of $\mathbf{e}_{k}, \nabla^{3} \mathbf{e}_{k}$, we get the following expression:

$$
\begin{gathered}
\nabla^{3} \mathbf{e}_{k}=\frac{1}{2}\left[\partial_{n} R_{m i k}^{l}+\partial_{n} P_{m i k}^{l}\right] \mathbf{e}_{l} d \xi^{n} d \xi^{m} d \xi^{i}+\frac{1}{2}\left[R_{m i k}^{l}+P_{m i k}^{l}\right] \frac{\partial \mathbf{e}_{l}}{\partial \xi^{n}} d \xi^{n} d \xi^{m} d \xi^{i} \\
+\frac{1}{2}\left[R_{m i k}^{l}+P_{m i k}^{l}\right] \mathbf{e}_{l}\left[d^{2} \xi^{m} d \xi^{i}+p d \xi^{m} d^{2} \xi^{i}+d \xi^{m} d^{2} \xi^{i}\right]+\Gamma_{i k}^{l} \mathbf{e}_{l} d^{3} \xi^{i}
\end{gathered}
$$

If we choose $p=j$ and $d^{3} \xi^{k}=0$, this complicated formula simplifies to:

$$
\begin{gathered}
\nabla^{3} \mathbf{e}_{k}=R_{m i k}^{l} \mathbf{e}_{l} d^{2} \xi^{m} d \xi^{i}+\frac{1}{2}\left[\nabla_{n} R_{i m k}^{l}-\nabla_{m} R_{i n k}^{l}\right] \mathbf{e}_{l} d \xi^{n} d \xi^{i} d \xi^{m} \\
+\frac{i \sqrt{3}}{2}\left[\nabla_{n} R_{i m k}^{l}+\nabla_{m} R_{i n k}^{l}\right] \mathbf{e}_{l} d \xi^{n} d \xi^{i} d \xi^{m}
\end{gathered}
$$

It is interesting to note that only two combinations of the covariant derivative of $R_{i k m}^{l}$ appear here; as a matter of fact, the third one, $\nabla_{i} R_{m n k}^{l}$ is linearly dependent by virtue of Bianchi identity.

The expression for $\nabla^{3} \mathbf{e}_{k}$ in the case of $Z_{3}$-graded differential calculus obtained above has also a clear geometrical meaning. In the usual ( $Z_{2}$-graded) case, the condition of vanishing of the expression $\nabla^{2} \mathbf{e}_{k}$ was equivalent with the zero-curvature condition, $R_{i m k}^{l}=0$; here, the vanishing of $\nabla^{3} \mathbf{e}_{k}$ also implies vanishing curvature, however, another invariant and interesting condition can be formulated, i.e.

$$
\nabla^{3} \mathbf{e}_{k}=R_{i m k}^{l} \mathbf{e}_{l}
$$

which implies constant curvature, the condition satisfied in symmetric spaces.

\section{A glimpse at possible future developments}

Classical gauge fields appear as the necessary device that maintains the covariance of the Dirac equation with respect to unitary transformations of the spinor wave function, $\psi \rightarrow e^{i S} \psi$ when $S$ becomes a function of the space-time coordinates. Then we replace the free 4 -momentum operator $p_{\mu}$ by its covariant counterpart $p_{\mu}-i e A_{\mu}$; simultaneously with a gauge transformation $\psi \rightarrow e^{i S} \psi$, we have (in the simplest, abelian case) $A_{\mu} \rightarrow A_{\mu}+\partial_{\mu} S$

It is natural to ask what is the generalization of Dirac's equation that would lead to the modified gauge theory, with curvature 3 -form $\Omega$ introduced above. The answer is quite obvious: if in the classical case the curvature 2 -form $F_{\mu \nu}$ containing the first derivatives of $A$ did appear naturally when we diagonalized the Dirac equation, applying once more the conjugate Dirac operator, here we 
must introduce a Schrödinger-like equation linear in the momentum operator, only the third power of which would become diagonal.

This leads naturally to a ternary generalization of Clifford algebras. Instead of the usual binary relation defining the usual Clifford algebra,

$$
\gamma^{\mu} \gamma^{\nu}+\gamma^{\nu} \gamma^{\mu}=2 g^{\mu \nu} \mathbf{1}, \quad \text { with } g^{\mu \nu}-g^{\nu \mu}=0
$$

we should introduce its ternary generalization, which is quite obvious (see also V. Abramov, [32]) :

$$
Q^{a} Q^{b} Q^{c}+Q^{b} Q^{c} Q^{a}+Q^{c} Q^{b} Q^{a}=3 \eta^{a b c} \mathbf{1}
$$

where the tensor $\eta^{a b c}$ must satisfy $\quad \eta^{a b c}=\eta^{b c a}=\eta^{c a b}$

The lowest-dimensional representation of such an algebra is given by complex $3 \times 3$ matrices:

$$
Q^{1}=\left(\begin{array}{ccc}
0 & 1 & 0 \\
0 & 0 & j \\
j^{2} & 0 & 0
\end{array}\right), \quad Q^{2}=\left(\begin{array}{ccc}
0 & 1 & 0 \\
0 & 0 & j^{2} \\
j & 0 & 0
\end{array}\right), \quad Q^{3}=\left(\begin{array}{ccc}
0 & 1 & 0 \\
0 & 0 & 1 \\
1 & 0 & 0
\end{array}\right)
$$

These matrices are given the $Z_{3}$-grade 1 ; their hermitian conjugates $Q^{* a}=\left(Q^{a}\right)^{\dagger}$ are of $Z_{3}$-grade 2 , whereas the diagonal matrices are of $Z_{3}$-grade 0 ; it is easy to verify that the so defined grades add up modulo 3.

The matrices $Q^{a}(a=1,2,3)$ satisfy the ternary relations (49) with $\eta^{a b c}$ a totally-symmetric tensor, whose only non-vanishing components are $\eta^{111}=\eta^{222}=\eta^{333}=1, \eta^{123}=\eta^{231}=\eta^{321}=j^{2}$, and $\eta^{321}=\eta^{213}=\eta^{132}=j$.

Therefore, the $Z_{3}$-graded generalization of Dirac's equation should read:

$$
\frac{\partial \psi}{\partial t}=Q^{1} \frac{\partial \psi}{\partial x}+Q^{2} \frac{\partial \psi}{\partial y}+Q^{3} \frac{\partial \psi}{\partial z}+B m \psi
$$

where $\psi$ stands for a triplet of wave functions, which can be considered either as a column, or as a grade 1 matrix with three non-vanishing entries $u v w$, and $B$ is the diagonal $3 \times 3$ matrix with the eigenvalues $1 j$ and $j^{2}$. It is interesting to note that this is possible only with three spatial coordinates.

In order to diagonalize this equation, we must act three times with the same operator, which will lead to the same equation of third order, satisfied by each of the three components $u, v, w$, e.g.:

$$
\frac{\partial^{3} u}{\partial t^{3}}=\left[\frac{\partial^{3}}{\partial x^{3}}+\frac{\partial^{3}}{\partial y^{3}}+\frac{\partial^{3}}{\partial z^{3}}-\frac{\partial^{3}}{\partial x \partial y \partial z}\right] u+m^{3} u
$$

This equation can be solved by separation of variables; the time-dependent and the space-dependent factors have the same structure:

$$
A_{1} e^{\omega t}+A_{2} e^{j \omega t}+A_{3} e^{j^{2} \omega t}, \quad B_{1} e^{\mathbf{k} . \mathbf{r}}+B_{2} e^{j \mathbf{k} \cdot \mathbf{r}}+B_{3} e^{j^{2} \mathbf{k} . \mathbf{r}}
$$


and their nine independent products can be represented in a basis of real functions as

$$
\left(\begin{array}{ccc}
A_{11} e^{\omega t+\mathbf{k} . \mathbf{r}} & A_{12} e^{\omega t-\frac{\mathbf{k} . \mathbf{r}}{2}} \cos \xi & A_{13} e^{\omega t-\frac{\mathbf{k} . \mathbf{r}}{2}} \sin \xi \\
A_{21} e^{-\frac{\omega t}{2}+\mathbf{k} \cdot \mathbf{r}} \cos \tau & A_{22} e^{-\frac{\omega t}{2}-\frac{\mathbf{k} . \mathbf{r}}{2}} \cos \tau \cos \xi & A_{23} e^{-\frac{\omega t}{2}-\frac{\mathbf{k} \cdot \mathbf{r}}{2}} \cos \tau \sin \xi \\
A_{31} e^{-\frac{\omega t}{2}+\mathbf{k} \cdot \mathbf{r}} \sin \tau & A_{32} e^{-\frac{\omega t}{2}-\frac{\mathbf{k} \cdot \mathbf{r}}{2}} \sin \tau \cos \xi & A_{33} e^{-\frac{\omega t}{2}-\frac{\mathbf{k} \cdot \mathbf{r}}{2}} \sin \tau \sin \xi
\end{array}\right)
$$

where $\tau=\frac{\sqrt{3}}{2} \omega t$ and $\xi=\frac{\sqrt{3}}{2} \mathbf{k r}$. The parameters $\omega, \mathbf{k}$ and $m$ must satisfy the cubic dispersion relation:

$$
\omega^{3}=k_{x}^{3}+k_{y}^{3}+k_{z}^{3}-3 k_{x} k_{y} k_{z}+m^{3}
$$

This relation is invariant under the simultaneous change of sign of $\omega, \mathbf{k}$ and $m$, which suggests the introduction of another set of solutions constructed in the same manner, but with minus sign in front of $\omega$ and $\mathbf{k}$, which we shall call conjugate solutions.

Although neither of these functions belongs to the space of tempered distributions, on which a Fourier transform can be performed, their ternary skewsymmetric products contain only trigonometric functions, depending on the combinations $2(\tau-\xi)$ and $2(\tau+\xi)$. As a matter of fact, not only the determinant, but also each of the minors of the above matrix is a combination of the trigonometric functions only. The same is true for the binary products of "conjugate" solutions, with the opposite signs of $\omega t$ and k.r in the exponentials.

This fact suggests that it is possible to obtain via linear combinations of these products the solutions of second or first order differential esuations, like Klein-Gordon or Dirac equation.

Still, the parameters $\omega$ and $\mathbf{k}$ do not satisfy the proper mass shell relations; however, it is possible to find new parameters, which are linear combinations of these, that will satisfy quadratic relations that may be intrpreted as a mass shell equation. We can more readily see this if we use the following parametrisation: let us put

$$
\begin{gathered}
\zeta=\left(k_{x}+k_{y}+k_{z}\right), \quad \chi=\operatorname{Re}\left(j k_{x}+j^{2} k_{y}+k_{z}\right), \quad \eta=\operatorname{Im}\left(j k_{x}+j^{2} k_{y}+k_{z}\right), \\
\text { and } \quad r^{2}=\chi^{2}+\eta^{2} \quad \phi=\operatorname{Arctg}(\eta / \chi) .
\end{gathered}
$$

In these coordinates the cubic mass hyperboloid equation becomes

$$
\omega^{3}-\zeta r^{2}=m^{3}
$$

Two obvious symmetries can be immediately seen here, the rotation around the axis $[1,1,1](\phi \rightarrow \phi+\delta \phi)$, and simultaneous dilatation of $\zeta$ and $r$ :

$$
r \rightarrow \lambda r, \quad \zeta \rightarrow \lambda^{-2} \zeta
$$

The same relation can be factorized as

$$
(\omega+\zeta)\left(\omega^{2}-r^{2}\right)+(\omega-\zeta)\left(\omega^{2}+r^{2}\right)=2 m^{3}
$$


We can define a one-dimensional subset of the above 3-dimensional hypersurface by requiering

$$
\omega^{2}-r^{2}=\left[2 m^{3}-(\omega-\zeta)\left(\omega^{2}+r^{2}\right)\right] /(\omega+\zeta)=M^{2}=\text { Const. }
$$

If we have three hypersurfaces (corresponding to the dispersion relations of three quarks satisfying the 3-rd order differential equation), which are embedded in the 12-dimensional space $M_{4} \times M_{4} \times M_{4}$, then the resulting 3-dimensional hypersurface defined by the above constrained applied to each of the three dispersion relations independently will produce the ordinary mass hyperboloid

$$
\omega_{1}^{2}+\omega_{2}^{2}+\omega_{3}^{2}-r_{1}^{2}-r_{2}^{2}-r_{3}^{2}=\Omega^{2}-r_{1}^{2}-r_{2}^{2}-r_{3}^{2}=3 M^{2}
$$

Another way to achieve a similar result is to observe that we need not multiply the solutions of our third-order differential equation pointwise, i.e. with the same argument; we should rather multiply the solutions with the same value of $t$, but with different values of $\mathbf{k}_{a}$ et $\mathbf{r}_{b}$. Then we must impose supplementary conditions on the parameters $\omega_{a}, \mathbf{k}_{a}$ and $\mathbf{r}_{c}$ in order to cancel all real exponentials in these products. In terms of these variables the resulting constraints amount to something very close to confinement, because our solutions will be subjected to the conditions of the general type

$$
\mathbf{k}_{1} \cdot \mathbf{r}_{1}-\frac{1}{2} \mathbf{k}_{2} \cdot \mathbf{r}_{2}-\frac{1}{2} \mathbf{k}_{3} \cdot \mathbf{r}_{3}=0
$$

which will at the same time factorize the cubic dispersion relation producing (although not in a unique way) a relativistic mass hyperboloid for certain linear cominations of $\omega_{a}$ and $\mathbf{k}_{b}$.

The solutions of our third-order differential equation do not belong to the space of tempered distributions and their Fourier transform is not well defined; also their products can not be represented as inverse Fourier transforms of the convolution of their Fourier transforms. Nevertheless, as in classical field theory, we can do this if their supports are restricted to positive frequencies only. Then one can write symbolically the convolution of three quark field propagators as follows:

$$
\frac{1}{\omega^{3}-\mathbf{k}^{3}-m^{3}} * \frac{1}{\omega^{3}-\mathbf{k}^{3}-m^{3}} * \frac{1}{\omega^{3}-\mathbf{k}^{3}-m^{3}}
$$

where $\mathbf{k}^{3}$ stands for the cubic form $\eta^{a b c} k_{a} k_{b} k_{c}$, and the integral is taken over the cubic hyperboloid (;); to the product of wave functions of quark with antiquark corresponds one convolution of two factors of this type.

According to our hypothesis, the convolution of the Fourier transforms of three quark (or anti-quark) propagators should generate the propagator of the corresponding composed particle, i.e. a fermion, whereas the convolution of two such propagators should give the propagator of a boson.

A simple power counting gives the dimension of the Fourier transform of the resulting propagator: 
$(-3) \times 3+2 \times D=-9+2 D$ in the first case, and $(-3) \times 2+D=-6+D$ in the second case, where $D$ is the dimension of the space- time. It is only when the dimension $D=4$ that we get the resulting propagator of dimension -1 for a ternary combination, and of dimension -2 for a binary combination, which is what is observed indeed for fermions and bosons, i.e. fields obeying the first and second order wave equations, respectively.

\section{Postscript}

It was intended that this paper dedicated to André Trautman be shorter than was eventually the case. The reason was that so much of my work, even including the most recent, can trace its genesis to the early influence of André. Knowing André's predilection for chess and chessboards, I hope that the patchwork of ideas presented above will provide him with an amusement of finding the resonances and echos of his own ideas.

Acknowledgements.

Numerous enlightening discussions with V.Abramov, B.Le Roy, L. Vainerman, M.Dubois-Violette, J.Madore, J.Lukierski and P.Kulish are gratefully acknowledged.

\section{References}

[1] J.W. Goethe, Faust, erster teil, (Goethes Werke, Abt.1. Bd.14.), Weimar, Hermann Böhlau ed. (1887); also: ed. Reclam, Stuttgart (1992)

[2] J.J. Rousseau, Julie ou la Nouvelle Héloise, édition présentée, établie et annotée par H. Coulet, Gallimard, Paris (1993).

[3] Ch. Fourier, Le socialisme sociétaire, (extrait des oeuvres complètes, Société nouvelle de librairie et d'édition, Paris (1903).

[4] K. Marx, Das Kapital, (1886)

[5] A. Lichnerowicz, Théorie des connexions et Groupes d'holonomie, Dunod, Paris, (1956)

[6] A. Trautman, Reports in Math.Physics, 1,(1971), 29

[7] A. Trautman, Differential Geometry for Physicists, Bibliopolis, Napoli, (1984)

[8] R. Kerner, Ann.Inst. H.Poincaré, 9 (2), (1968), 147

[9] R. Kerner, Ann.Inst. H.Poincaré, 34(4), (1981), 437

[10] Y.M. Cho and P.G.O. Freund, Phys. Rev. D 12 (1975), 1711 
[11] E. Witten, Nucl. Phys. B 186, (1981), 412

[12] R. Kerner, Journ. of Math. Physics, 24 (2) (1983), 356

[13] R. Kerner, CERN preprint TH 3669 (1983)

[14] Ch. Bertrand, R. Kerner, Lett.Math.Phys.,18 (1989), 193

[15] Ch. Bertrand, R. Kerner, S. Mignemi, Int.Journ.Mod.Phys. A, 7 (31), (1992), 7741

[16] I. Newton, Principia edited in: Sir Isaac Newton's Mathematical Principles of Natural Philosophy and His System of the World, Andrew Motte, trans. London; reprint: Greenwood, New-York (1969)

[17] M. Dubois-Violette, R. Kerner, J. Madore, Journ.Math.Phys., 33, (1990), 312

[18] M. Dubois-Violette, R. Kerner, J. Madore, Journ.Math.Phys., 33, (1990), 323

[19] M. Dubois-Violette, J. Madore, R. Kerner, Class. and Quantum Gravity, 8, (1991), 1077

[20] A. Connes, J. Lott, Nuclear Physics B (Proc.Suppl), 18 (1990), 29

[21] R. Coquereaux, G. Esposito-Farèse, C. Vaillant, Nucl.Phys. B 353, (1991), 689

[22] J. Madore, Phys.Lett.A (1992)

[23] A. H. Chamseddine, G. Felder, J. Frölich, Phys. Lett B, 296, (1992), 109

[24] F. Scheck, Physics Lett. B 284 (1992), 303

[25] R. Kerner, Comptes Rendus Acad. Sci. Paris, Sér II, t.312, (1991), p.191 $-196$.

[26] R. Kerner, Journ. Math.Phys. 33 (1992), p.403 - 411.

[27] R. Lawrence, "Algebras and triangle relations", in Topological Methods in Field Theory, ed. by J. Mickelsson and O. Pekoneti, World Scientific, (1992), p. $429-447$.

[28] L. Vainerman, R. Kerner, Journ. Math. Phys., to appear (1996)

[29] Won-Sang Chung, Journ. Math. Phys. 35(5), (1994), 2497

[30] R. Kerner, in "Generalized Symmetries in Physics", p. 375-394, H.D. Doebner, V. Dobrev and A. Ushveridze eds., World Scientific (1994). 
[31] B. Le Roy, Journ. of Math. Physics, 35 (1), (1995), 474

[32] V. Abramov, Algebras, Groups and Geometries, 12 (1995), 201 